Check for updates

Cite this: RSC Adv., 2021, 11, 33235

Received 5th July 2021

Accepted 28th September 2021

DOI: $10.1039 / \mathrm{d} 1 \mathrm{ra05187c}$

rsc.li/rsc-advances

\title{
Consecutive reactions to construct tricarbonyl compounds and synthetic applications thereof $\dagger$
}

\author{
Diego Madroñero, (D) a Cesar A. Mujica-Martinez (iD ${ }^{\mathrm{b}}$ and Alfredo Vázquez (D) *a \\ Lithium anions derived from $\mathrm{O}$-carbonate-protected cyanohydrins undergo conjugate addition to \\ cycloalkenones with the concomitant transfer of the alkoxycarbonyl group to produce tricarbonyl \\ compounds. These products offer numerous possibilities for further elaboration. The synthetic potential \\ of the cascade products was demonstrated by forming bicyclic and tricyclic systems through \\ intramolecular condensation reactions.
}

\section{Introduction}

Since the pioneering work by Stork et al., ${ }^{1}$ O-protected cyanohydrins have become recognized as valuable acyl anion synthons. ${ }^{2,3}$ Over the years, this approach has been widely used ${ }^{4 a-k}$ to achieve the chemical synthesis of organic molecules with diverse architectures (Scheme 1).

Among the different groups used to protect the hydroxyl group in cyanohydrins, carbonates ${ }^{5 a-c}$ display a moderately electrophilic carbon atom. This reactivity can be exploited to perform subsequent transformations to the anions' reaction with electrophiles (i.e., transfer of an acyl group), thus enabling consecutive reactions. Consecutive reactions, also known as cascade or domino reactions ${ }^{6}$ are a practical strategy to form multiple bonds sequentially, simplifying the construction of organic molecules.

A recent paper $^{7}$ demonstrated that anions derived from Ocarbonate-protected cyanohydrins undergo conjugate addition to cyclohexenone with concomitant transfer of the alkoxycarbonyl group to produce $\beta$-keto- $\beta$-acylcycloalkanecarboxylic acid esters; however, these were isolated as the enol acetate derivatives.

Tricarbonyl compounds have been used for the synthesis of functionalized biphenyls via an oxidative aromatization with iodine $^{8 a}$ and for the formal synthesis of $\left( \pm\right.$ )-cochlearol ${ }^{8} .^{8 b}$ The oxidation of tricarbonyl compounds has been used for the preparation of propellanes, compounds showing a broad spectra of biological and pharmacological activities, ${ }^{8 c}$ as well as for the synthesis of heterocycles. ${ }^{8 d}$

${ }^{a}$ Departamento de Química Orgánica, Facultad de Química, Universidad Nacional Autónoma de México, Ciudad Universitaria, Cd. Mx., 04510, Mexico. E-mail: joseavm@unam.mx

${ }^{b}$ GIFBA, Departamento de Química, Facultad de Ciencias Exactas y Naturales, Centro de Investigación en Materiales CIMA, Universidad de Nariño, San Juan de Pasto, 520002, Colombia

$\dagger$ Electronic supplementary information (ESI) available. See DOI: $10.1039 / \mathrm{d} 1 \mathrm{ra05187 \textrm {c }}$
Considering the tremendous synthetic utility of the functionalities present in the putative intermediate products namely $\beta$-keto ester, ${ }^{9 a-f}$ gamma-keto ester, ${ }^{9 g}$ and 1,4 -dione, ${ }^{10}$ we attempted to directly obtain these intermediates by modifying Le Lagadec's procedure. ${ }^{7}$

Herein, we present a procedure to construct synthetically valuable tricarbonyl cyclic compounds featuring consecutive Michael-Claisen reactions of lithium anions derived from $\mathrm{O}-$

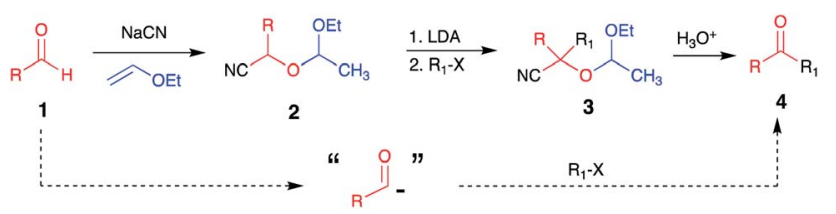

Scheme 1 Pioneering work of Stork and Maldonado to use protected cyanohydrins as acyl anion equivalents.

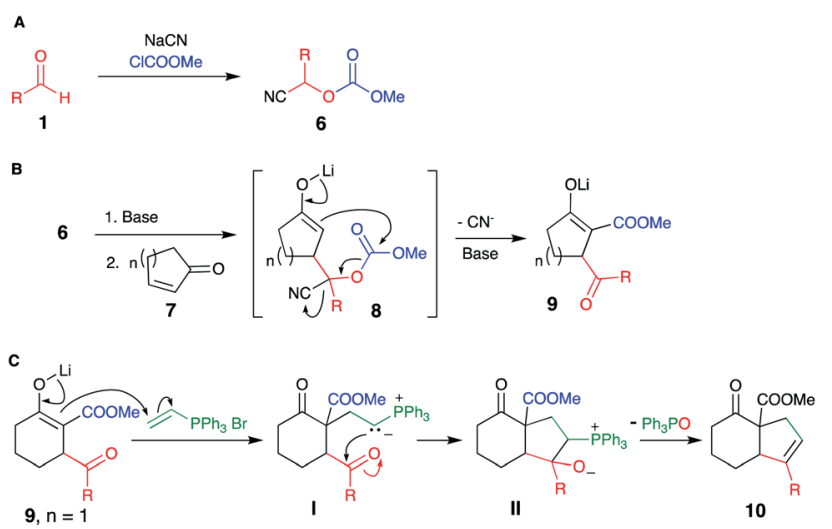

Scheme 2 (A and B) Synthetic strategy for the construction of tricarbonyl compounds using consecutive reactions. (B and C) Plausible mechanism for the consecutive reactions illustrated for the formation of 10 . 
carbonate-protected cyanohydrins with 5, 6, and 7-membered cycloalkenones. The functionalities introduced into the cycloalkenones was exploited by annulation of a five-membered ring onto the olefin of the original cycloalkenone.

\section{Results and discussion}

Our strategy is shown in Scheme 2, consisting of the preparation of cyanocarbonates $\mathbf{6}$ from aldehydes $\mathbf{1}$ according to literature procedures (Scheme 2A). Deprotonation with a suitable base ( 2 equiv.), followed by conjugate addition of the corresponding anions onto cyclic enones 7 (Scheme 2B) and subsequent reaction of the tricarbonyl product $\mathbf{9}$ (as an enolate) with electrophiles would afford highly functionalized products $\mathbf{1 0}$ (Scheme 2C).

The preparation of some cyanocarbonates 6 was achieved using the two-phase reaction system procedure reported by Kolis et al. $^{\mathbf{1 1}}$ (Table 1, Method A). In other cases, higher yields were obtained using ethyl carbonocyanidate (CNCOOEt) in the

Table 1 Preparation of cyanocarbonates

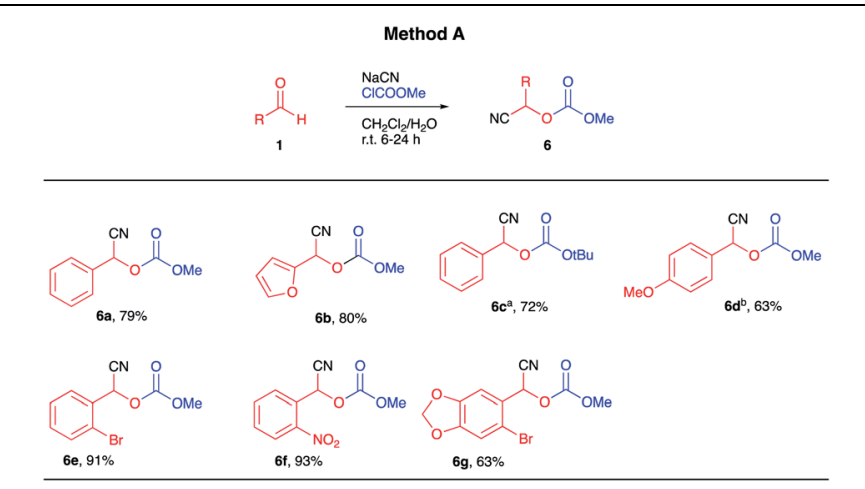

${ }^{\mathrm{a}}(\mathrm{Boc})_{2} \mathrm{O}$ was used instead of $\mathrm{ClCOOMe},{ }^{\mathrm{b}}$ The reaction was heated at $40^{\circ} \mathrm{C}$.

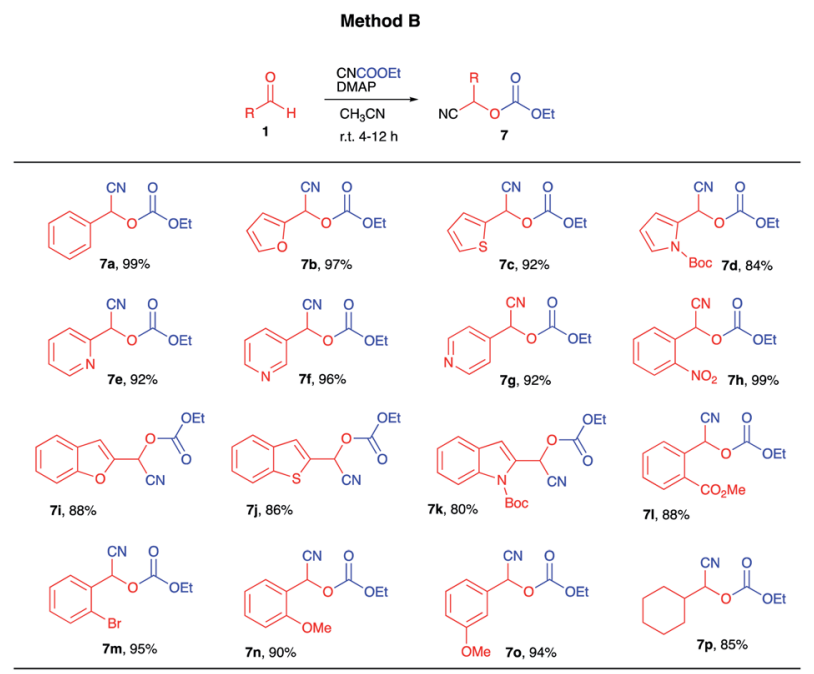

${ }^{a}$ (Boc) $)_{2} \mathrm{O}$ was used instead of ClCOOMe. ${ }^{b}$ The reaction was heated at $40{ }^{\circ} \mathrm{C}$. presence of DMAP and $\mathrm{CH}_{3} \mathrm{CN}$ as the solvent (Table 1, Method B). ${ }^{5 c}$ A total of 23 cyanocarbonates were prepared and satisfactorily characterized.

To evaluate the deprotonation ease of the NC-C-H bond, its $\mathrm{p} K_{\mathrm{a}}$ was determined using a direct approach, ${ }^{\mathbf{1 2}}$ in which free energies are calculated directly in THF solution at $-78^{\circ} \mathrm{C}$ (Table 2). Calculations were carried out using the $\omega$ B97XD DFT hybrid functional, ${ }^{13}$ the $6-311++\mathrm{G}(\mathrm{d}, \mathrm{p})$ basis set, and the D2 Grimme dispersion correction ${ }^{\mathbf{1 4}}$ as implemented in the Gaussian 16 suite. ${ }^{15}$ The solvent was described implicitly using the SMD method. ${ }^{16}$ This methodology has been used before to determine $\mathrm{p} K_{\mathrm{a}}$ in several systems. ${ }^{12,17}$ Fig. 1 shows that the obtained $\mathrm{p} K_{\mathrm{a}}$ values highly correlate with the Hammett constants ${ }^{18}(\sigma)$ of the phenyl substituent for compounds $\mathbf{6 a}, \mathbf{6 d}, \mathbf{6 e}$, and $\mathbf{6 f}$, for which it was obtained $\mathrm{p} K_{\mathrm{a}}=37.503-11.527 \sigma$ and $R^{2}=0.931$. Therefore, the deprotonation ease of these compounds increases with the electron-withdrawing properties of the substituents, which results in the stabilization of the corresponding carbanion. Similar correlations are observed with other molecular parameters and also for compounds 7 . The relatively large $\mathrm{p} K_{\mathrm{a}}$ values indicated the use of a strong base would be required to obtain the corresponding carbanion efficiently.

To test our hypothesis and standardize the reaction conditions, we selected cyanocarbonate $\mathbf{6 a}$ as the carbanion source and commercially available LiHMDS as the base. The base was added dropwise to a solution of $6 \mathbf{a}$ in THF at $-78{ }^{\circ} \mathrm{C}$. After $15 \mathrm{~min}$, cyclohex-2-en-1-one was added, and the course of the reaction was monitored by TLC.

Table 2 Scope of the cascade reactions between cyanocarbonates 6 and 7 with 2-cyclohexen-1-one

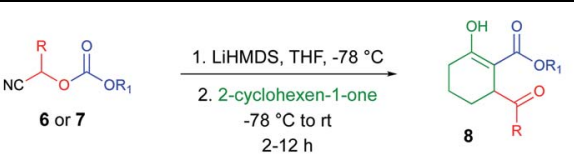

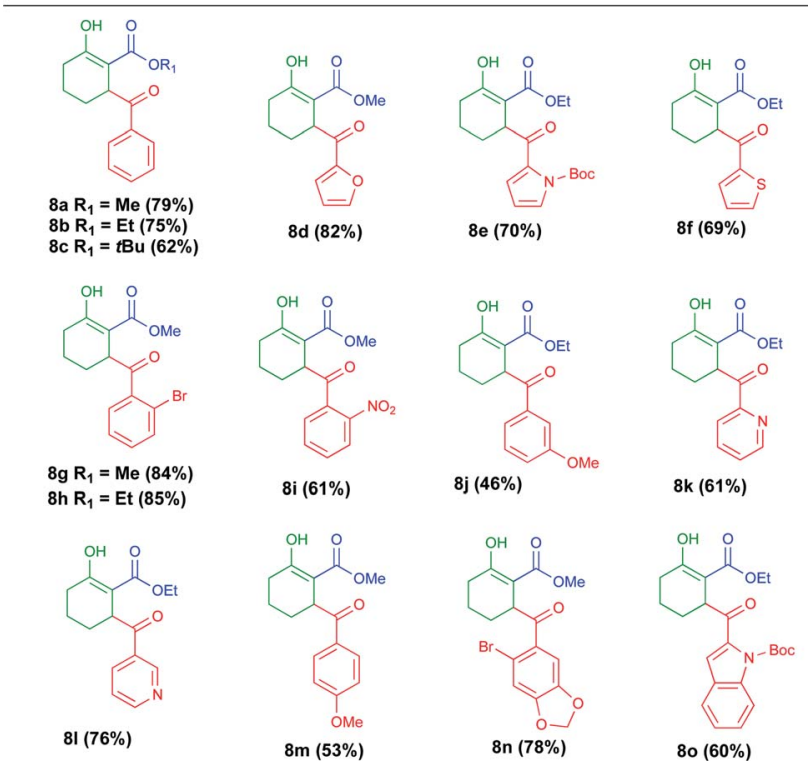




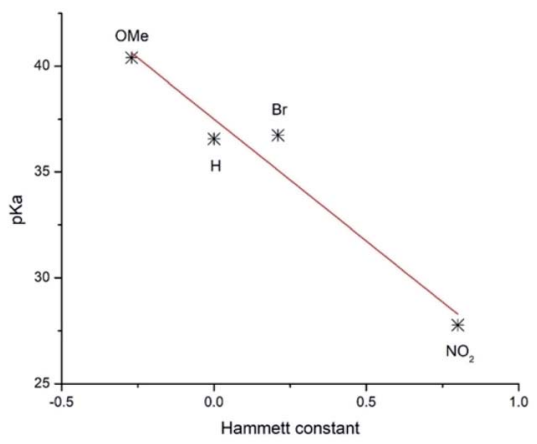

Fig. 1 Correlation of $\mathrm{p} K_{\mathrm{a}}$ with the Hammett constant of the phenyl substituent for compounds $6 \mathrm{a}, 6 \mathrm{~d}, 6 \mathrm{e}, 6 \mathrm{f}$.

To investigate the scope of the cascade process under the optimized conditions described above, the anions of cyanocarbonates 6 and 7 were added to cyclohexanone. The results of those experiments are summarized in Table 2. A total 15 of tricarbonyl compounds $(\mathbf{8 a}-\mathbf{o}$ and $\mathbf{9 a}-\mathbf{c})$ were obtained in moderate yields. All the products were successfully characterized.

Next, we investigated the use of 2-cyclopenten-1-one, 2cyclohepten-1-one, and 3-methyl-2-cyclohexen-1-one as the enone component for the cascade process to further explore the scope of the methodology. The results are presented in Table 3. For 2-cylopenten-1-one, the tricarbonyl compound 9a was obtained in $80 \%$ yield, whereas for 2-cyclohepten-1-one the desired product $9 \mathrm{~b}$ was obtained in $50 \%$ yield. In the case of $3-$ methyl-2-cyclohexen-1-one, 9c was obtained in 38\% yield. This lower yield was attributed to the steric hindrance caused by the methyl group at C-3 of the cycloalkenone. This assumption is supported by the failure to obtain the cascade product when the more sterically hindered 4,4-dimethyl-2-cyclohexen-1-one was used as the substrate.

Interestingly, ${ }^{1} \mathrm{H}$ NMR spectra for all the cascade products obtained from 2-cyclohexen-1-one and 3-methyl-2-cyclohexen-1one were isolated as a mixture of keto and enol tautomers. In contrast only a single keto tautomer or the keto diastereomers

Table 3 Use of different cycloalkenones to explore the scope of the process
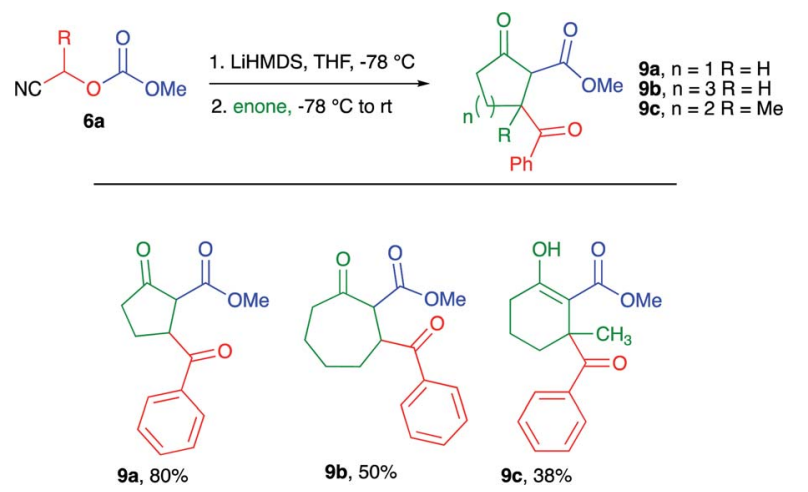

$9 b, 50 \%$

$9 c, 38 \%$ were observed by ${ }^{1} \mathrm{H}$ NMR for the adducts derived from 2cyclopenten-1-one and 2-cyclohepten-1-one (i.e., 9a and 9b). Computational results indicate that the keto-tautomer of compounds 9a and $9 \mathrm{~b}$ is 1.365 and $1.366 \mathrm{kcal} \mathrm{mol}^{-1}$, respectively, more stable than the corresponding enol-tautomer. On the contrary, for compound $\mathbf{8 a}$, the enol-tautomer is only $0.274 \mathrm{kcal} \mathrm{mol}^{-1}$ more stable than the corresponding ketotautomer. This small energy difference could explain the reason to observe this product as a mixture of tautomers. Further investigation on the tautomeric behavior of these systems is currently underway. Clearly, the cascade process can occur for 5, 6, and 7-membered cycloalkenones, even enones showing moderate steric hindrance.

After proving the efficiency of the cascade process, we investigated the possibility of performing annulations via intramolecular condensation reactions. Thus, when $14 \mathbf{b}$ was treated with $\mathrm{NaH}$ in THF at rt, followed by the addition of triphenylvinylphosphonium bromide (Schweizer's reagent) ${ }^{19}$ no reaction was observed. If the reaction mixture was heated under reflux, several spots are observed on TLC. However, when DBU was used as the base (in $\mathrm{CH}_{3} \mathrm{CN}$ ), $39 \%$ of intramolecular Wittig product 10 was obtained after purification, along with unreacted starting material and traces of two unknown compounds. Treatment of $\mathbf{8 b}$ with methylvinyl ketone and cyclopentenone as the Michael acceptors in the presence of $\mathrm{DBU}$ (in $\mathrm{CH}_{3} \mathrm{CN}$ at room temperature), afforded cyclic products 11 and 12 in 88 and $32 \%$ yield, respectively (Table 4 ). It is noteworthy that decarboxylation occurred during the formation of $\mathbf{1 1}$ and 12.

The preparation of annulated products 10, 11, and 12 nicely exemplifies the synthetic potential of $\beta$-keto- $\beta$-acylcycloalkanecarboxylic acid esters $\mathbf{8}$ as scaffolds to obtain products with increased structural complexity. With some adjustments, we believe that compounds 8 can be used to obtain diverse molecules such as the core of pacifigorgianes ${ }^{\mathbf{2 0}} \mathbf{1 3}$, the

Table 4 Further transformations of cascade product $8 \mathrm{~b}$

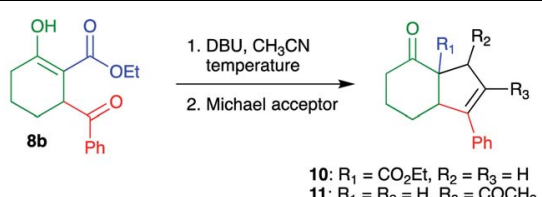

10: $\mathrm{R}_{1}=\mathrm{CO}_{2} \mathrm{Et}, \mathrm{R}_{2}=\mathrm{R}_{3}=\mathrm{H}$

11: $R_{1}=R_{2}=H, R_{3}=\mathrm{COCH}_{3}$
12: $\mathrm{R}_{2}=\mathrm{CH}_{2} \mathrm{CH}_{2}, \mathrm{R}=\mathrm{CO}$

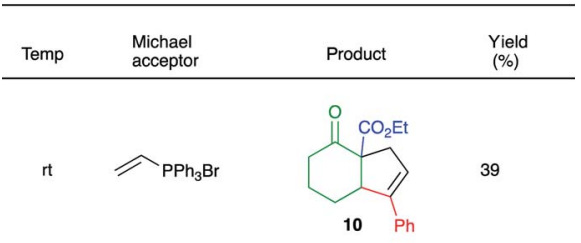

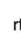
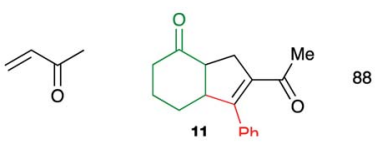

Reflux
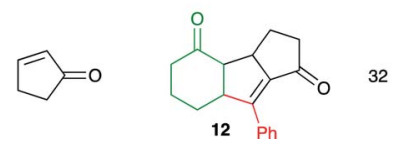

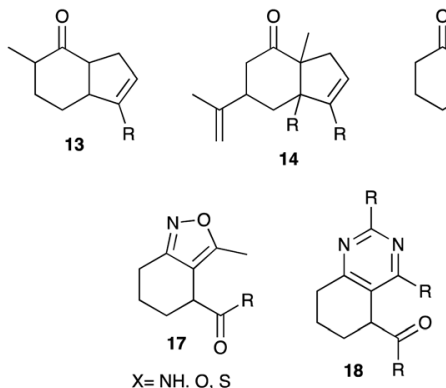

15
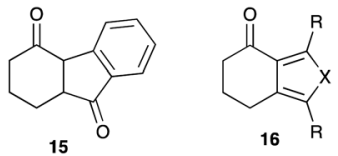

$\mathrm{X}=\mathrm{NH} . \mathrm{O}, \mathrm{S}$

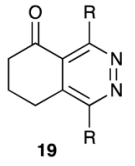

Fig. 2 Potential use of tricarbonyl compounds 8 to generate structural diversity.

sesquiterpenoid cyperolone ${ }^{21} 14$, indanones ${ }^{22} 15$, furans ${ }^{23}$ pyrroles and thiophenes 16, 1,2-azoles 17, pyrimidines 18 and 1,2-diazines 19 (Fig. 2).

\section{Experimental}

\section{General information}

All experiments involving air and/or moisture-sensitive compounds were conducted in an oven dried round-bottom flask capped with a rubber septum under a positive pressure of nitrogen. Air- and moisture-sensitive liquids were transferred via syringe or stainless-steel cannula. Low temperature baths were ice/water $\left(0^{\circ} \mathrm{C}\right), \mathrm{CO}_{2}(\mathrm{~s}) /$ acetone $\left(-78^{\circ} \mathrm{C}\right)$. Unless otherwise noted, reaction temperatures refer to that of the bath. Concentration refers to removal of volatiles on a rotary evaporator below $35{ }^{\circ} \mathrm{C}$. Analytical thin-layer chromatography (TLC) was performed using glass plates pre-coated with silica gel $(0.25$ $\mathrm{mm}, 60 \AA$ pore size, $230-400$ mesh, Merck) impregnated with a fluorescent indicator $(254 \mathrm{~nm})$. Materials on TLC plates were visualized under an ultraviolet lamp $(254 \mathrm{~nm})$ and/or by submersion of the plate in a solution of phosphomolybdic acid (5\%) containing a trace of ceric sulfate in aqueous sulfuric acid $(5 \% \mathrm{v} / \mathrm{v})$ followed by charring on a hot plate. Flash column chromatography (FCC) was performed according to Still et al. with silica gel $60(40-63 \mu \mathrm{m})$. All mixed solvent eluents are reported as $\mathrm{v} / \mathrm{v}$ solutions. Unless otherwise noted, all reported compounds were homogeneous by thin layer chromatography (TLC) and by ${ }^{1} \mathrm{H}$ NMR.

\section{Materials and methods}

All the reagents were purchased from Sigma-Aldrich and were used as received unless other thing stated. Solvents were distilled prior to use. Anhydrous solvents were distilled under nitrogen atmosphere. THF and diethyl ether were distilled on sodium benzophenone ketyl; $\mathrm{MeOH}$ on magnesium activated with $5 \%$ iodine. $\mathrm{Et}_{3} \mathrm{~N}, \mathrm{CH}_{3} \mathrm{CN}$ dichloroethane and DMF were distilled on $\mathrm{CaH}_{2}$.

Proton nuclear magnetic resonance $\left({ }^{1} \mathrm{H}\right.$ NMR) spectra and carbon nuclear magnetic resonance $\left({ }^{13} \mathrm{C} \mathrm{NMR}\right)$ spectra were recorded on Agilent-Inova-300 and Varian VNMRS-400 NMR spectrometers. Proton chemical shifts are expressed in parts per million ( $\mathrm{ppm}, \delta$ scale) and are referenced to tetramethylsilane
(TMS: 0.0). Carbon chemical shifts are expressed in parts per million (ppm, $\delta$ scale) and are referenced to tetramethylsilane (TMS: 0.0 ). For compounds $6 \mathbf{6 e}, \mathbf{7 e}, \mathbf{7 f}, \mathbf{7 h}, \mathbf{7 p}, \mathbf{8 g}$, and $\mathbf{8 n}, \mathrm{CDCl}_{3}$ was used as a standard. This information is included in the ESI. $\dagger$ Data are represented as follows: chemical shift, multiplicity $(\mathrm{s}=$ singlet, $\mathrm{d}=$ doublet, $\mathrm{t}=$ triplet, $\mathrm{q}=$ quartet, $\mathrm{dd}=$ doublet of doublets, $\mathrm{dt}=$ doublet of triplets, $\mathrm{td}=$ triplet of doublets, $\mathrm{ddd}=$ doublet of doublets of doublets, $\mathrm{ddt}=$ doublet of doublets of triplets, dqd = doublet of quartets of doublets, $\mathrm{m}$ $=$ multiplet, $\mathrm{br}=$ broad, app $=$ apparent), integration, and coupling constant $(J)$ in Hertz (Hz).

IR spectra were recorded on a PerkinElmer Spectrum 400 FTIR/FIR spectrometer with ATR. Mass spectra were carried out on a JEOL SMX-102a spectrometer.

\section{Method A}

A $2 \mathrm{M}$ aqueous solution of $\mathrm{NaCN}(4.8 \mathrm{~mL})$ was added dropwise to a mixture of the aldehyde ( $5 \mathrm{mmol})$, ClCOOMe $(0.43 \mathrm{~mL}, 5.5$ $\mathrm{mmol})$ and $(n \mathrm{Bu})_{4} \mathrm{NBr}(160 \mathrm{mg}, 0.5 \mathrm{mmol})$ in $\mathrm{CH}_{2} \mathrm{Cl}_{2}(6.25 \mathrm{~mL})$ at ambient temperature. The resultant mixture was vigorously stirred overnight, the two phases were separated, and the organic layer was washed with sat. $\mathrm{NaCl}$ dried on anhydrous $\mathrm{Na}_{2} \mathrm{SO}_{4}$ and concentrated in vacuo. The residue was purified by column chromatography $\left(\mathrm{SiO}_{2}\right)$.

\section{Method B}

CNCOOEt ( $0.108 \mathrm{~mL}, 1.1 \mathrm{mmol})$ was slowly added to a mixture of aldehyde ( $1 \mathrm{mmol})$ and DMAP $(6 \mathrm{mg}, 0.05 \mathrm{mmol})$ in $\mathrm{CH}_{3} \mathrm{CN}(2$ $\mathrm{mL}$ ) under nitrogen atmosphere at ambient temperature. When all the starting material has been consumed (TLC) the reaction mixture is poured on sat $\mathrm{NaCl}(5 \mathrm{~mL})$ and extracted with EtOAc $(3 \times 20 \mathrm{~mL})$. The organic extracts were combined and dried on anhydrous $\mathrm{Na}_{2} \mathrm{SO}_{4}$, the solvent evaporated in vacuo and the residue purified by column chromatography.

Cyano(phenyl)methyl methyl carbonate (6a). Prepared following Method A. The residue was purified by column chromatography (hexanes/EtOAc 95 : 5) to obtain $755 \mathrm{mg}$ (79\%) of a translucent yellow oil. ${ }^{1} \mathrm{H}$ NMR $\left(400 \mathrm{MHz}, \mathrm{CDCl}_{3}\right) \delta: 3.87(\mathrm{~s}$, $\left.3 \mathrm{H}, \mathrm{OCH}_{3}\right), 6.27(\mathrm{~s}, 1 \mathrm{H}, \mathrm{CHCN}), 7.47-7.48\left(\mathrm{~m}, 3 \mathrm{H}, m, p-\mathrm{C}_{6} \mathrm{H}_{5}\right)$, 7.53-7.56 (m, 2H, $\left.o-\mathrm{C}_{6} \mathrm{H}_{5}\right) \mathrm{ppm} .{ }^{13} \mathrm{C}$ NMR $\left(400 \mathrm{MHz}, \mathrm{CDCl}_{3}\right) \delta$ : 56.0, 66.7, 115.8, 128.0, 129.4, 130.8, 131.3, 154.2 ppm. APCI: $\mathrm{m} /$ $z$ calculated for $\mathrm{C}_{8} \mathrm{H}_{8} \mathrm{NO}_{4}[\mathrm{M}+\mathrm{H}]^{+}=192.0661$; found: 192.0703 .

Cyano(furan-2-yl)methyl methyl carbonate (6b). Prepared following Method A. The residue was purified by column chromatography (hexanes/EtOAc $95: 5)$ to obtain $729 \mathrm{mg}(80 \%)$ of a yellow oil. ${ }^{1} \mathrm{H}$ NMR $\left(400 \mathrm{MHz}, \mathrm{CDCl}_{3}\right) \delta: 3.86\left(\mathrm{~s}, 3 \mathrm{H}, \mathrm{OCH}_{3}\right)$, $6.34(\mathrm{~s}, 1 \mathrm{H}, \mathrm{CHCN}), 6.44-6.45\left(\mathrm{dd}, 1 \mathrm{H}, J=1.8,3.2 \mathrm{~Hz}, 3-\mathrm{C}_{4} \mathrm{H}_{3} \mathrm{O}\right)$, 6.72-6.73 (d, $\left.1 \mathrm{H}, J=3.2 \mathrm{~Hz}, 4-\mathrm{C}_{4} \mathrm{H}_{3} \mathrm{O}\right), 7.51-7.52(\mathrm{~d}, 1 \mathrm{H}, J=$ $\left.1.8 \mathrm{~Hz}, 5-\mathrm{C}_{4} \mathrm{H}_{3} \mathrm{O}\right) .{ }^{13} \mathrm{C}$ NMR $\left(400 \mathrm{MHz}, \mathrm{CDCl}_{3}\right) \delta: 56.1,59.5$, $111.3,113.3,113.8,143.6,145.4,154.0 \mathrm{ppm}$. APCI: $\mathrm{m} / \mathrm{z}$ calculated for $\mathrm{C}_{8} \mathrm{H}_{8} \mathrm{NO}_{4}[\mathrm{M}+\mathrm{H}]^{+}=182.0453$; found: 182.0502 .

tert-Butyl (cyano(phenyl)methyl)carbonate (6c). A 2 M aqueous solution of $\mathrm{NaCN}(4.8 \mathrm{~mL})$ was added dropwise to a mixture of benzaldehyde ( $5 \mathrm{mmol}), \mathrm{Boc}_{2} \mathrm{O}(1.2 \mathrm{~g}, 5.5 \mathrm{mmol})$ and $(n \mathrm{Bu})_{4} \mathrm{NBr}(16 \mathrm{mg}, 0.05 \mathrm{mmol})$ in $\mathrm{CH}_{2} \mathrm{Cl}_{2}(6.25 \mathrm{~mL})$ at ambient temperature. The resulting mixture was stirred 
overnight, the two phases were separated, and the organic layer was washed with sat $\mathrm{NaCl}$, dried over anhydrous $\mathrm{Na}_{2} \mathrm{SO}_{4}$ and concentrated in vacuo. The residue was purified by column chromatography (hexanes/EtOAc $80: 20$ ) to obtain $845 \mathrm{mg}$ (72\%) of a colorless oil. ${ }^{1} \mathrm{H}$ NMR $\left(400 \mathrm{MHz}, \mathrm{CDCl}_{3}\right) \delta: 1.44(\mathrm{~s}, 9 \mathrm{H}$, $\mathrm{O} t-\mathrm{Bu}), 6.14(\mathrm{~s}, 1 \mathrm{H}, \mathrm{CHCN}), 7.34-7.36\left(\mathrm{~m}, 3 \mathrm{H}, m, p-\mathrm{C}_{6} \mathrm{H}_{5}\right), 7.43-$ $7.45\left(\mathrm{~m}, 2 \mathrm{H}, o-\mathrm{C}_{6} \mathrm{H}_{5}\right) \mathrm{ppm} .{ }^{13} \mathrm{C} \mathrm{NMR}\left(400 \mathrm{MHz}, \mathrm{CDCl}_{3}\right) \delta: 27.7$, 65.8, 85.0, 116.2 , 128.0, 129.3, 130.6, 131.7, 151.7 ppm. APCI: $m /$ $z$ calculated for $\mathrm{C}_{13} \mathrm{H}_{16} \mathrm{NO}_{3}[\mathrm{M}+\mathrm{H}]^{+}=234.1130$; found: 234.1124 .

Cyano(4-methoxyphenyl)methyl methyl carbonate (6d). Prepared according to Method A with the following modification: the reaction mixture was stirred at $40{ }^{\circ} \mathrm{C}$ for $12 \mathrm{~h}$. The residue was purified by column chromatography (hexanes/ EtOAc $80: 20)$ to obtain $700 \mathrm{mg}(63 \%)$ of a pale-yellow oil. ${ }^{1} \mathrm{H}$ NMR (400 MHz, $\left.\mathrm{CDCl}_{3}\right) \delta: 3.82\left(\mathrm{~s}, 3 \mathrm{H}, \mathrm{COOCH}_{3}\right), 3.84(\mathrm{~s}, 3 \mathrm{H}$, $\left.\mathrm{OCH}_{3}\right), 6.20$ (s, 1H, CHCN), 6.95 (m, 2H m-C $\left.\mathrm{C}_{6} \mathrm{H}_{4} \mathrm{OMe}\right), 7.47$ (m, $\left.2 \mathrm{H}, o-\mathrm{C}_{6} \mathrm{H}_{4} \mathrm{OMe}\right) \mathrm{ppm} .{ }^{13} \mathrm{C} \mathrm{NMR}\left(400 \mathrm{MHz}, \mathrm{CDCl}_{3}\right) \delta: 55.5,55.9$, $66.5,114.7,116.0,123.4,129.9,154.2,161.5$ ppm. APCI: $m / z$ calculated for $\mathrm{C}_{11} \mathrm{H}_{12} \mathrm{NO}_{4}[\mathrm{M}+\mathrm{H}]^{+}=222.0766$; found: 222.0769 .

(2-Bromophenyl)(cyano)methyl methyl carbonate (6e). Prepared following Method A. The residue was purified by column chromatography (hexanes/EtOAc $90: 10$ ) to obtain $1.23 \mathrm{~g}(91 \%)$ of a colorless oil. ${ }^{1} \mathrm{H} \mathrm{NMR}\left(400 \mathrm{MHz}, \mathrm{CDCl}_{3}\right) \delta: 3.89$ $\left(\mathrm{s}, 3 \mathrm{H}, \mathrm{OCH}_{3}\right), 6.57$ (s, 1H, CHCN), 7.61-7.64 (m, $1 \mathrm{H}, m-\mathrm{C}_{6} \mathrm{H}_{4} \mathrm{Br}$ ), 7.73-7.76 (m, $\left.1 \mathrm{H}, m-\mathrm{C}_{6} \mathrm{H}_{4} \mathrm{Br}\right), 7.87-7.89$ (m, $\left.1 \mathrm{H}, p-\mathrm{C}_{6} \mathrm{H}_{4} \mathrm{Br}\right)$, 8.14$7.16\left(\mathrm{~m}, 1 \mathrm{H}, o-\mathrm{C}_{6} \mathrm{H}_{4} \mathrm{Br}\right) \mathrm{ppm} .{ }^{13} \mathrm{C} \mathrm{NMR}\left(400 \mathrm{MHz}, \mathrm{CDCl}_{3}\right) \delta: 56.4$, 63.0, 114.7, 126.0, 126.9, 129.3, 131.6, 134.9, 146.9, 153.6 ppm. ESI $^{+}: m / z$ calculated for $\mathrm{C}_{10} \mathrm{H}_{9} \mathrm{BrNO}_{3}[\mathrm{M}+\mathrm{H}]^{+}=269.9766$, found: 269.9775 .

Cyano(2-nitrophenyl)methyl methyl carbonate (6f). Prepared following Method A. The residue was purified by column chromatography (hexanes/EtOAc 90 : 10) to obtain $1.16 \mathrm{~g}(93 \%)$ of a colorless oil. ${ }^{1} \mathrm{H}$ NMR $\left(400 \mathrm{MHz} \mathrm{CDCl}_{3}\right) \delta: 3.89(\mathrm{~s}, 3 \mathrm{H}$, $\left.\mathrm{OCH}_{3}\right), 7.01(\mathrm{~s}, 1 \mathrm{H}, \mathrm{CHCN}), 7.67-7.71\left(\mathrm{~m}, 1 \mathrm{H}, m-\mathrm{C}_{6} \mathrm{H}_{4} \mathrm{NO}_{2}\right)$, 7.79-7.83 (m, $\left.1 \mathrm{H}, m-\mathrm{C}_{6} \mathrm{H}_{4} \mathrm{NO}_{2}\right)$, 7.94-7.96 (m, $1 \mathrm{H}, p-\mathrm{C}_{6} \mathrm{H}_{4} \mathrm{NO}_{2}$ ), 8.21-8.24 (m, $\left.1 \mathrm{H}, o-\mathrm{C}_{6} \mathrm{H}_{4} \mathrm{NO}_{2}\right)$ ppm. ${ }^{13} \mathrm{C} \mathrm{NMR}\left(400 \mathrm{MHz}, \mathrm{CDCl}_{3}\right.$ ) $\delta: 56.4,63.0,114.7,126.0 .127 .0,129.3,131.7,134.9,146.9$, 153.6 ppm. ESI $^{+}: m / z$ calculated for $\mathrm{C}_{10} \mathrm{H}_{9} \mathrm{~N}_{2} \mathrm{O}_{5}[\mathrm{M}+\mathrm{H}]^{+}=$ 237.0511, found: 237.0510 .

(6-Bromobenzo[d][1,3]dioxol-5-yl)(cyano)methyl methyl carbonate (6g). Prepared according to Method A with the following modification: the reaction mixture was stirred at $40{ }^{\circ} \mathrm{C}$ for $12 \mathrm{~h}$. The residue was purified by column chromatography (hexanes/EtOAc $80: 20)$ to obtain $1.32 \mathrm{~g}$ (84\%) of a white solid. ${ }^{1} \mathrm{H}$ NMR $\left(400 \mathrm{MHz}, \mathrm{CDCl}_{3}\right) \delta: 3.88\left(\mathrm{~s}, 3 \mathrm{H}, \mathrm{OCH}_{3}\right), 6.04(\mathrm{~s}, 2 \mathrm{H}$, $\left.\mathrm{OCH}_{2} \mathrm{O}\right), 6.51$ (s, $\left.1 \mathrm{H}, \mathrm{CHCN}\right), 7.04\left(\mathrm{~s}, 1 \mathrm{H}, m-\mathrm{C}_{6} \mathrm{H}_{2} \mathrm{Br}\right), 7.16(\mathrm{~s}, 1 \mathrm{H}$, $o-\mathrm{C}_{6} \mathrm{H}_{2} \mathrm{Br}$ ) ppm. ${ }^{13} \mathrm{C} \mathrm{NMR}\left(400 \mathrm{MHz}, \mathrm{CDCl}_{3}\right) \delta: 56.2,66.2,102.8$, 109.3, 113.3, 114.9, 115.3, 123.8, 148.3, 150.5, 153.9 ppm. ESI ${ }^{+}$: $m / z$ calculated for $\mathrm{C}_{11} \mathrm{H}_{9} \mathrm{BrNO}_{5}[\mathrm{M}+\mathrm{H}]^{+}=313.9664$, found: 313.9658 .

Cyano(phenyl)methyl ethyl carbonate (7a). Prepared according to Method B (reaction time $8 \mathrm{~h}$ ). The residue was purified by column chromatography (hexanes/EtOAc $80: 20$ ) to obtain $203 \mathrm{mg}$ (99\%) of a colorless oil. ${ }^{1} \mathrm{H}$ NMR $(400 \mathrm{MHz}$, $\left.\mathrm{CDCl}_{3}\right) \delta: 1.32\left(\mathrm{t}, 3 \mathrm{H}, J=7.2 \mathrm{~Hz}, \mathrm{OCH}_{2} \mathrm{CH}_{3}\right), 4.21-4.33(\mathrm{~m}, 2 \mathrm{H}$, $\left.\mathrm{OCH}_{2} \mathrm{CH}_{3}\right), 6.26(\mathrm{~s}, 1 \mathrm{H}, \mathrm{CHCN}), 7.44-7.45\left(\mathrm{~m}, 3 \mathrm{H}, m, p-\mathrm{C}_{6} \mathrm{H}_{5}\right)$,
7.52-7.54 (m, 2H, o- $\left.\mathrm{C}_{6} \mathrm{H}_{5}\right)$ ppm. ${ }^{13} \mathrm{C} \mathrm{NMR}\left(400 \mathrm{MHz}, \mathrm{CDCl}_{3}\right) \delta$ : 14.2, 65.7, 66.5, 115.9, 128.0, 129.4, 130.7, 131.4, 153.5 ppm. APCI: $m / z$ calculated for $\mathrm{C}_{11} \mathrm{H}_{12} \mathrm{NO}_{3}[\mathrm{M}+\mathrm{H}]^{+}=206.0817$; found: 206.0849 .

Cyano(furan-2-yl)methyl ethyl carbonate (7b). Prepared according to Method B (reaction time $8 \mathrm{~h}$ ). The residue was purified by column chromatography (hexanes/EtOAc $95: 5$ ) to obtain $190 \mathrm{mg}$ (97\%) of a translucent yellow oil. ${ }^{1} \mathrm{H}$ NMR (400 $\left.\mathrm{MHz}, \mathrm{CDCl}_{3}\right) \delta: 1.32\left(\mathrm{t}, 3 \mathrm{H}, J=7.1 \mathrm{~Hz}, \mathrm{OCH}_{2} \mathrm{CH}_{3}\right), 4.22-4.33(\mathrm{~m}$, $2 \mathrm{H}, \mathrm{OCH}_{2} \mathrm{CH}_{3}$ ), 6.32 (s, $\left.1 \mathrm{H}, \mathrm{CHCN}\right), 6.43(\mathrm{dd}, 1 \mathrm{H}, J=1.8,3.2 \mathrm{~Hz}$, $\left.3-\mathrm{C}_{4} \mathrm{H}_{3} \mathrm{O}\right), 6.71\left(\mathrm{~d}, 1 \mathrm{H}, J=3.2 \mathrm{~Hz}, 4-\mathrm{C}_{4} \mathrm{H}_{3} \mathrm{O}\right), 7.5(\mathrm{~d}, 1 \mathrm{H}, J=$ $\left.1.8 \mathrm{~Hz}, 5-\mathrm{C}_{4} \mathrm{H}_{3} \mathrm{O}\right) \mathrm{ppm} .{ }^{13} \mathrm{C} \mathrm{NMR}\left(400 \mathrm{MHz}, \mathrm{CDCl}_{3}\right) \delta: 14.2,59.3$, $65.9,111.3,113.1,113.9,143.8,145.4,153.3$ ppm. APCI: $m / z$ calculated for $\mathrm{C}_{9} \mathrm{H}_{10} \mathrm{NO}_{4}[\mathrm{M}+\mathrm{H}]^{+}=196.0610$; found: 196.0626 .

Cyano(thiophen-2-yl)methyl ethyl carbonate (7c). Prepared according to Method B (reaction time $6 \mathrm{~h}$ ). The residue was purified by column chromatography (hexanes/EtOAc $80: 20$ ) to obtain $195 \mathrm{mg}(92 \%)$ of a brown oil. ${ }^{1} \mathrm{H}$ NMR (400 $\mathrm{MHz}, \mathrm{CDCl}_{3}$ ) $\delta: 1.33\left(\mathrm{t}, 3 \mathrm{H}, J=7.16 \mathrm{~Hz}, \mathrm{OCH}_{2} \mathrm{CH}_{3}\right), 4.25-4.32(\mathrm{~m}, 2 \mathrm{H}$, $\mathrm{OCH}_{2} \mathrm{CH}_{3}$ ), 6.48 (s, 1H, CHCN), 7.04-7.05 (m, 1H, 4- $\left.\mathrm{C}_{4} \mathrm{H}_{3} \mathrm{~S}\right)$, 7.36-7.38 (m, $\left.1 \mathrm{H}, 3-\mathrm{C}_{4} \mathrm{H}_{3} \mathrm{~S}\right), 7.46-7.47\left(\mathrm{~m}, 1 \mathrm{H}, 5-\mathrm{C}_{4} \mathrm{H}_{3} \mathrm{~S}\right) \mathrm{ppm}$. ${ }^{13} \mathrm{C}$ NMR (400 MHz, $\mathrm{CDCl}_{3}$ ) $\delta: 14.1,61.5,65.8,115.0,127.3$, $129.4,130.0,132.7,153.2 \mathrm{ppm}$. APCI: $\mathrm{m} / \mathrm{z}$ calculated for $\mathrm{C}_{9} \mathrm{H}_{10} \mathrm{NO}_{3} \mathrm{~S}[\mathrm{M}+\mathrm{H}]^{+}=212.0381$; found: 212.0395 .

tert-Butyl 2-(cyano((ethoxycarbonyl)oxy)methyl)-1H-pyrrole1-carboxylate (7d). Prepared according to Method B (reaction time $12 \mathrm{~h}$ ). The residue was purified by column chromatography (hexanes/EtOAc $70: 30$ ) to obtain $248 \mathrm{mg}$ (84\%) of a translucent orange oil ${ }^{1} \mathrm{H}$ NMR (400 MHz, $\left.\mathrm{CDCl}_{3}\right) \delta: 1.33(\mathrm{t}, 3 \mathrm{H}, J=7.13 \mathrm{~Hz}$, $\mathrm{OCH}_{2} \mathrm{CH}_{3}$ ), 1.58 (s, 9H, Ot-Bu), 4.22-4.33 (m, 2H, $\mathrm{OCH}_{2} \mathrm{CH}_{3}$ ), 6.18-6.20 (t, $\left.1 \mathrm{H}, J=3.4 \mathrm{~Hz}, 4-\mathrm{C}_{4} \mathrm{H}_{3} \mathrm{~N}\right), 6.70-6.71(\mathrm{~m}, 1 \mathrm{H}, 3-$ $\left.\mathrm{C}_{4} \mathrm{H}_{3} \mathrm{~N}\right), 6.81(\mathrm{~s}, 1 \mathrm{H}, \mathrm{CHCN}), 7.31-7.32\left(\mathrm{~m}, 1 \mathrm{H}, 5-\mathrm{C}_{4} \mathrm{H}_{3} \mathrm{~N}\right) \mathrm{ppm}$. ${ }^{13} \mathrm{C} \mathrm{NMR}\left(400 \mathrm{MHz}, \mathrm{CDCl}_{3}\right.$ ) $\delta: 14.3,28.0,60.3$, 65.5, 85.7, 110.7, $115.4,117.6,123.5,124.7,148.4,153.5$ ppm. APCI: $m / z$ calculated for $\mathrm{C}_{14} \mathrm{H}_{19} \mathrm{~N}_{2} \mathrm{O}_{5}[\mathrm{M}+\mathrm{H}]^{+}=295.1294$; found: 295.1214.

Cyano(pyridin-2-yl)methyl ethyl carbonate (7e). Prepared according to Method B (reaction time $12 \mathrm{~h}$ ). The residue was purified by column chromatography (hexanes/EtOAc $80: 20$ ) to obtain $190 \mathrm{mg}$ (92\%) of a translucent yellow oil. ${ }^{1} \mathrm{H}$ NMR (400 $\left.\mathrm{MHz}, \mathrm{CDCl}_{3}\right) \delta: 1.35\left(\mathrm{t}, 3 \mathrm{H}, J=7.15 \mathrm{~Hz}, \mathrm{OCH}_{2} \mathrm{CH}_{3}\right), 4.27-4.35$ (m, 2H, $\left.\mathrm{OCH}_{2} \mathrm{CH}_{3}\right), 6.37$ (s, 1H, CHCN), 7.37-7.39 (m, 1H, 5$\left.\mathrm{C}_{5} \mathrm{H}_{4} \mathrm{~N}\right)$, 7.58-7.60 (m, 1H, 3- $\left.\mathrm{C}_{5} \mathrm{H}_{4} \mathrm{~N}\right), 7.80-7.83(\mathrm{~m}, 1 \mathrm{H}, 4-$ $\left.\mathrm{C}_{5} \mathrm{H}_{4} \mathrm{~N}\right), 8.66-8.67\left(\mathrm{~m}, 1 \mathrm{H}, 6-\mathrm{C}_{5} \mathrm{H}_{4} \mathrm{~N}\right) \mathrm{ppm} .{ }^{13} \mathrm{C} \mathrm{NMR}(400 \mathrm{MHz}$, $\left.\mathrm{CDCl}_{3}\right) \delta: 14.2,66.0,67.2,115.3,121.9,125.0,137.8,150.3$, 150.7, 153.4 ppm. APCI: $m / z$ calculated for $\mathrm{C}_{13} \mathrm{H}_{14} \mathrm{NO}_{5}[\mathrm{M}+\mathrm{H}]^{+}$ $=207.0770$, found: 207.0797.

Cyano(pyridin-3-yl)methyl ethyl carbonate (7f). Prepared according to Method B (reaction time $12 \mathrm{~h}$ ). The residue was purified by column chromatography (hexanes/EtOAc $90: 10$ ) to obtain $197 \mathrm{mg}$ (96\%) of a translucent yellow oil. ${ }^{1} \mathrm{H}$ NMR (400 $\left.\mathrm{MHz}, \mathrm{CDCl}_{3}\right) \delta: 1.32\left(\mathrm{t}, 3 \mathrm{H}, J=7.13 \mathrm{~Hz}, \mathrm{OCH}_{2} \mathrm{CH}_{3}\right), 4.23-4.33$ $\left(\mathrm{m}, 2 \mathrm{H}, \mathrm{OCH}_{2} \mathrm{CH}_{3}\right), 6.30(\mathrm{~s}, 1 \mathrm{H}, \mathrm{CHCN}), 7.40-7.42(\mathrm{~m}, 1 \mathrm{H}, 5-$ $\left.\mathrm{C}_{5} \mathrm{H}_{4} \mathrm{~N}\right), 7.89-7.91\left(\mathrm{~m}, 1 \mathrm{H}, 4-\mathrm{C}_{5} \mathrm{H}_{4} \mathrm{~N}\right), 8.70-8.71(\mathrm{~m}, 1 \mathrm{H}, 6-$ $\left.\mathrm{C}_{5} \mathrm{H}_{4} \mathrm{~N}\right), 8.76-8.77\left(\mathrm{~m}, 1 \mathrm{H}, 2-\mathrm{C}_{5} \mathrm{H}_{4} \mathrm{~N}\right) \mathrm{ppm} .{ }^{13} \mathrm{C} \mathrm{NMR}(400 \mathrm{MHz}$, $\left.\mathrm{CDCl}_{3}\right) \delta: 14.2,64.3,66.1,115.1,124.1,127.6,135.6,149.2$, 152.0, 153.3 ppm. APCI: $m / z$ calculated for $\mathrm{C}_{13} \mathrm{H}_{14} \mathrm{NO}_{5}[\mathrm{M}+\mathrm{H}]^{+}$ $=207.0770$, found: 207.0797 . 
Cyano(pyridin-4-yl)methyl ethyl carbonate (7g). Prepared according to Method B (reaction time $12 \mathrm{~h}$ ). The residue was purified by column chromatography (hexanes/EtOAc $80: 20$ ) to obtain $190 \mathrm{mg}$ (92\%) of a translucent yellow oil. ${ }^{1} \mathrm{H}$ NMR (400 $\mathrm{MHz}, \mathrm{CDCl} 3) \delta: 1.29\left(\mathrm{t}, 3 \mathrm{H}, J=7.14 \mathrm{~Hz}, \mathrm{OCH}_{2} \mathrm{CH}_{3}\right), 4.22-4.29$ $\left(\mathrm{m}, 2 \mathrm{H}, \mathrm{OCH}_{2} \mathrm{CH}_{3}\right), 6.22(\mathrm{~s}, 1 \mathrm{H}, \mathrm{CHCN}), 7.40(\mathrm{~d}, 2 \mathrm{H}, J=6.19 \mathrm{~Hz}$, $\left.3,5-\mathrm{C}_{5} \mathrm{H}_{4} \mathrm{~N}\right), 8.68\left(\mathrm{~d}, 2 \mathrm{H}, J=6.19 \mathrm{~Hz}, 2,6-\mathrm{C}_{5} \mathrm{H}_{4} \mathrm{~N}\right) \mathrm{ppm} .{ }^{13} \mathrm{C} \mathrm{NMR}$ $\left(400 \mathrm{MHz}, \mathrm{CDCl}_{3}\right) \delta: 14.2,64.8,66.2,114.8,121.6,139.8,150.9$, 153.3 ppm. APCI: $m / z$ calculated for $\mathrm{C}_{13} \mathrm{H}_{14} \mathrm{NO}_{5}[\mathrm{M}+\mathrm{H}]^{+}=$ 207.0770, found: 207.0809 .

Cyano(2-nitrophenyl)methyl ethyl carbonate (7h). Prepared according to Method B (reaction time $2 \mathrm{~h}$ ). The residue was purified by column chromatography (hexanes/EtOAc $90: 10$ ) to obtain $245 \mathrm{mg}$ (98\%) of colorless oil. ${ }^{1} \mathrm{H}$ NMR (400 MHz, $\mathrm{CDCl}_{3}$ ) $\delta: 1.35\left(\mathrm{t}, 3 \mathrm{H}, J=7.15 \mathrm{~Hz}, \mathrm{OCH}_{2} \mathrm{CH}_{3}\right), 4.26-4.34(\mathrm{~m}, 2 \mathrm{H}$, $\mathrm{OCH}_{2} \mathrm{CH}_{3}$ ), 7.00 (s, $\left.1 \mathrm{H}, \mathrm{CHCN}\right), 7.67-7.70\left(\mathrm{~m}, 1 \mathrm{H}, m-\mathrm{C}_{6} \mathrm{H}_{4} \mathrm{NO}_{2}\right.$ ), 7.79-7.83 (m, $\left.1 \mathrm{H}, m-\mathrm{C}_{6} \mathrm{H}_{4} \mathrm{NO}_{2}\right)$, 7.94-7.96 (m, $1 \mathrm{H}, p-\mathrm{C}_{6} \mathrm{H}_{4} \mathrm{NO}_{2}$ ), 8.21-8.23 (m, 1H, o- $\left.\mathrm{C}_{6} \mathrm{H}_{4} \mathrm{NO}_{2}\right)$ ppm. ${ }^{13} \mathrm{C} \mathrm{NMR} \mathrm{(400} \mathrm{MHz,} \mathrm{CDCl}_{3}$ ) $\delta: 14.2,62.8,66.2,114.8,126.0,127.1,129.3,131.6,134.9,146.9$, 153.0 ppm. APCI: $m / z$ calculated for $\mathrm{C}_{11} \mathrm{H}_{11} \mathrm{~N}_{2} \mathrm{O}_{5}[\mathrm{M}+\mathrm{H}]^{+}=$ 251.0668; found: 251.0599.

Benzofuran-2-yl(cyano)methyl ethyl carbonate (7i). Prepared according to Method B (reaction time $5 \mathrm{~h}$ ). The residue was purified by column chromatography (hexanes/EtOAc $80: 20$ ) to obtain $216 \mathrm{mg}(88 \%)$ of a translucent yellow oil. ${ }^{1} \mathrm{H}$ NMR $(400$ $\left.\mathrm{MHz}, \mathrm{CDCl}_{3}\right) \delta: 1.36\left(\mathrm{t}, 3 \mathrm{H}, J=7.15 \mathrm{~Hz}, \mathrm{OCH}_{2} \mathrm{CH}_{3}\right), 4.29-4.37$ $\left(\mathrm{m}, 2 \mathrm{H}, \mathrm{OCH}_{2} \mathrm{CH}_{3}\right), 6.49(\mathrm{~s}, 1 \mathrm{H}, \mathrm{CHCN}), 7.10\left(\mathrm{~s}, 1 \mathrm{H}, 3-\mathrm{C}_{8} \mathrm{H}_{5} \mathrm{O}\right)$, 7.28-7.32 (m, 1H, 6- $\left.\mathrm{C}_{8} \mathrm{H}_{5} \mathrm{O}\right), 7.38-7.42\left(\mathrm{~m}, 1 \mathrm{H}, 5-\mathrm{C}_{8} \mathrm{H}_{5} \mathrm{O}\right), 7.52-$ $7.54\left(\mathrm{~m}, 1 \mathrm{H}, 7-\mathrm{C}_{8} \mathrm{H}_{5} \mathrm{O}\right), 7.62-7.64\left(\mathrm{~m}, 1 \mathrm{H}, 4-\mathrm{C}_{8} \mathrm{H}_{5} \mathrm{O}\right) \mathrm{ppm} .{ }^{13} \mathrm{C}$ NMR (400 MHz, $\left.\mathrm{CDCl}_{3}\right) \delta: 14.2,59.9,66.1,109.5,112.0,113.7$, 122.2, 123.9, 126.6, 127.0, 145.8, 153.3, 155.9 ppm. APCI: $m / z$ calculated for $\mathrm{C}_{13} \mathrm{H}_{12} \mathrm{NO}_{4}[\mathrm{M}+\mathrm{H}]^{+}=246.0766$, found: 246.0770 .

Benzo $[\boldsymbol{b}]$ thiophen-2-yl(cyano)methyl ethyl carbonate (7j). Prepared according to Method B (reaction time $6 \mathrm{~h}$ ). The residue was purified by column chromatography (hexanes/EtOAc 90 : 10) to obtain $226 \mathrm{mg}(86 \%)$ of a translucent yellow oil. ${ }^{1} \mathrm{H}$ NMR (400 MHz, $\left.\mathrm{CDCl}_{3}\right) \delta: 1.36\left(\mathrm{t}, 3 \mathrm{H}, J=7.14 \mathrm{~Hz}, \mathrm{OCH}_{2} \mathrm{CH}_{3}\right)$, 4.28-4.36 (m, 2H, $\left.\mathrm{OCH}_{2} \mathrm{CH}_{3}\right), 6.58(\mathrm{~s}, 1 \mathrm{H}, \mathrm{CHCN}), 7.41-7.44(\mathrm{~m}$, $\left.2 \mathrm{H}, 5,6-\mathrm{C}_{8} \mathrm{H}_{5} \mathrm{~S}\right), 7.62-7.63\left(\mathrm{~m}, 1 \mathrm{H}, 3-\mathrm{C}_{8} \mathrm{H}_{5} \mathrm{~S}\right), 7.81-7.86(\mathrm{~m}, 2 \mathrm{H}$, 4,7- $\mathrm{C}_{8} \mathrm{H}_{5} \mathrm{~S}$ ) ppm. ${ }^{13} \mathrm{C} \mathrm{NMR}\left(400 \mathrm{MHz}, \mathrm{CDCl}_{3}\right) \delta: 14.2,62.4,66.0$, $114.8,122.7,124.8,125.2$, 126.2, 126.8, 133.3, 138.4, 140.9, 153.3 ppm. APCI: $m / z$ calculated for $\mathrm{C}_{13} \mathrm{H}_{13} \mathrm{NO}_{4}[\mathrm{M}-\mathrm{OEt}]^{+}=$ 216.0119, found: 216.0106.

tert-Butyl 2-(cyano((ethoxycarbonyl)oxy)methyl)-1H-indole1-carboxylate (7k). Prepared according to Method B (reaction time $6 \mathrm{~h}$ ). The residue was purified by column chromatography (hexanes/EtOAc $70: 30)$ to obtain $275 \mathrm{mg}(80 \%)$ of a translucent yellow oil. ${ }^{1} \mathrm{H}$ NMR $\left(400 \mathrm{MHz}, \mathrm{CDCl}_{3}\right) \delta: 1.36(\mathrm{t}, 3 \mathrm{H}, J=7.10 \mathrm{~Hz}$, $\mathrm{OCH}_{2} \mathrm{CH}_{3}$ ), 1.70 (s, 9H, Ot-Bu), 4.30-4.35 (m, 2H, $\mathrm{OCH}_{2} \mathrm{CH}_{3}$ ), 6.99 (s, 1H, CHCN), 7.08-7.09 (m, 1H, 3- $\left.\mathrm{C}_{8} \mathrm{H}_{5} \mathrm{~N}\right), 7.25-7.29(\mathrm{~m}$, $\left.1 \mathrm{H}, 5-\mathrm{C}_{8} \mathrm{H}_{5} \mathrm{~N}\right)$, 7.35-7.39 (m, 1H, 6- $\left.\mathrm{C}_{8} \mathrm{H}_{5} \mathrm{~N}\right)$, 7.57-7.59 (m, 1H, 4$\left.\mathrm{C}_{8} \mathrm{H}_{5} \mathrm{~N}\right), 8.06-8.09\left(\mathrm{~m}, 1 \mathrm{H}, 7-\mathrm{C}_{8} \mathrm{H}_{5} \mathrm{~N}\right) \mathrm{ppm} .{ }^{13} \mathrm{C}$ NMR $(400 \mathrm{MHz}$, $\left.\mathrm{CDCl}_{3}\right) \delta: 14.3$, 28.2, 61.6, 65.7, 86.1, 112.6, 115.3, 116.0, 121.7, 123.6, 126.2, 127.8, 129.3, 137.0, 149.8, 153.4 ppm. APCI: $m / z$ calculated for $\mathrm{C}_{18} \mathrm{H}_{21} \mathrm{~N}_{2} \mathrm{O}_{5}[\mathrm{M}+\mathrm{H}]^{+}=345.1450$, found: 245.1482 .
Methyl 2-(cyano((ethoxycarbonyl)oxy)methyl)benzoate (7l). Prepared according to Method B (reaction time $6 \mathrm{~h}$ ). The residue was purified by column chromatography (hexanes/EtOAc 90 : 10) to obtain $232 \mathrm{mg}$ (88\%) of a translucent yellow oil. ${ }^{1} \mathrm{H}$ NMR (400 MHz, $\mathrm{CDCl}_{3}$ ) $\delta: 1.33\left(\mathrm{t}, J=7.11 \mathrm{~Hz}, 3 \mathrm{H}, \mathrm{OCH}_{2} \mathrm{CH}_{3}\right.$ ), 3.93 (s, 3H, OMe), 4.25-4.33 (m, 2H, $\left.\mathrm{OCH}_{2} \mathrm{CH}_{3}\right), 7.28(\mathrm{~s}, 1 \mathrm{H}$, CHCN), 7.51-7.55 (m, $\left.1 \mathrm{H}, m-\mathrm{C}_{6} \mathrm{H}_{4} \mathrm{CO}_{2} \mathrm{Me}\right), 7.63-7.67$ (m, $1 \mathrm{H} \mathrm{m}$ $\mathrm{C}_{6} \mathrm{H}_{4} \mathrm{CO}_{2} \mathrm{Me}$ ), 7.83-7.86 (m, $1 \mathrm{H}, p-\mathrm{C}_{6} \mathrm{H}_{4} \mathrm{CO}_{2} \mathrm{Me}$ ), 8.05-8.10 (m, $\left.1 \mathrm{H}, o-\mathrm{C}_{6} \mathrm{H}_{4} \mathrm{CO}_{2} \mathrm{Me}\right) \mathrm{ppm} .{ }^{13} \mathrm{C} \mathrm{NMR}\left(400 \mathrm{MHz}, \mathrm{CDCl}_{3}\right) \delta: 14.1$, 52.7, 63.6, 65.6, 115.9, 128.1, 128.2, 130.2, 131.5, 132.8, 133.4, 153.2, 166.2 ppm. APCI: $m / z$ calculated for $\mathrm{C}_{13} \mathrm{H}_{14} \mathrm{NO}_{5}[\mathrm{M}+\mathrm{H}]^{+}$ $=264.0872$, found: 264.0841 .

(2-Bromophenyl)(cyano)methyl ethyl carbonate $(7 \mathrm{~m})$. Prepared according to Method B (reaction time $6 \mathrm{~h}$ ). The residue was purified by column chromatography (hexanes/EtOAc $80: 20)$ to obtain $270 \mathrm{mg}(95 \%)$ of translucent oil. ${ }^{1} \mathrm{H}$ NMR $\left(400 \mathrm{MHz}, \mathrm{CDCl}_{3}\right) \delta: 1.34\left(\mathrm{t}, 3 \mathrm{H}, J=7.15 \mathrm{~Hz}, \mathrm{OCH}_{2} \mathrm{CH}_{3}\right), 4.27-$ $4.33\left(\mathrm{~m}, 2 \mathrm{H}, \mathrm{OCH}_{2} \mathrm{CH}_{3}\right), 6.57(\mathrm{~s}, 1 \mathrm{H}, \mathrm{CHCN}), 7.30-7.34(\mathrm{~m}, 1 \mathrm{H}$, $m-\mathrm{C}_{6} \mathrm{H}_{4} \mathrm{Br}$ ), 7.40-7.45 (m, $\left.1 \mathrm{H}, m-\mathrm{C}_{6} \mathrm{H}_{4} \mathrm{Br}\right)$, 7.61-7.64 (m, $1 \mathrm{H}, p$ $\left.\mathrm{C}_{6} \mathrm{H}_{4} \mathrm{Br}\right), 7.71-7.74\left(\mathrm{~m}, 1 \mathrm{H}, o-\mathrm{C}_{6} \mathrm{H}_{4} \mathrm{Br}\right) \mathrm{ppm} .{ }^{13} \mathrm{C} \mathrm{NMR}(400 \mathrm{MHz}$, $\left.\mathrm{CDCl}_{3}\right) \delta: 14.1,65.8(\times 2), 115.1,123.1,128.3,129.6,130.7,132.0$, 133.5, 153.1 ppm. ESI $^{+}: m / z$ calculated for $\mathrm{C}_{11} \mathrm{H}_{11} \mathrm{BrNO}_{3}[\mathrm{M}+\mathrm{H}]^{+}$ $=283.9922$, found: 283.9927 .

Cyano(2-methoxyphenyl)methyl ethyl carbonate (7n). Prepared according to Method B (reaction time $12 \mathrm{~h}$ ). The residue was purified by column chromatography (hexanes/ EtOAc $90: 10)$ to obtain $212 \mathrm{mg}(90 \%)$ of a translucent yellow oil. ${ }^{1} \mathrm{H}$ NMR (400 MHz, $\left.\mathrm{CDCl}_{3}\right) \delta: 1.33(\mathrm{t}, J=7.12 \mathrm{~Hz}, 3 \mathrm{H}$, $\mathrm{OCH}_{2} \mathrm{CH}_{3}$ ), 3.88 (s, 3H, OMe), 4.24-4.32 (m, 2H, $\mathrm{OCH}_{2} \mathrm{CH}_{3}$ ), $6.58(\mathrm{~s}, 1 \mathrm{H}, \mathrm{CHCN}), 6.92-6.95\left(\mathrm{~m}, 1 \mathrm{H}, o-\mathrm{C}_{6} \mathrm{H}_{4} \mathrm{OMe}\right), 7.00-7.05$ (m, $1 \mathrm{H}, p-\mathrm{C}_{6} \mathrm{H}_{4} \mathrm{OMe}$ ), 7.40-7.44 (m, 1H, $m-\mathrm{C}_{6} \mathrm{H}_{4} \mathrm{OMe}$ ), 7.56-7.58 (m, $1 \mathrm{H} m-\mathrm{C}_{6} \mathrm{H}_{4} \mathrm{OMe}$ ) ppm. ${ }^{13} \mathrm{C} \mathrm{NMR}\left(400 \mathrm{MHz}, \mathrm{CDCl}_{3}\right) \delta: 14.1$, 55.7, 61.7, 65.4, 111.1, 115.9, 119.5, 120.9, 128.9, 132.0, 153.5, 156.7 ppm. APCI: $m / z$ calculated for $\mathrm{C}_{12} \mathrm{H}_{14} \mathrm{NO}_{4}[\mathrm{M}+\mathrm{H}]^{+}=$ 236.0923, found: 236.0930 .

Cyano(3-methoxyphenyl)methyl ethyl carbonate (7o). Prepared according to Method B (reaction time $12 \mathrm{~h}$ ). The residue was purified by column chromatography (hexanes/ EtOAc $90: 10)$ to obtain $222 \mathrm{mg}(94 \%)$ of a translucent yellow oil. ${ }^{1} \mathrm{H}$ NMR (400 MHz, $\left.\mathrm{CDCl}_{3}\right) \delta: 1.33(\mathrm{t}, J=7.12 \mathrm{~Hz}, 3 \mathrm{H}$, $\mathrm{OCH}_{2} \mathrm{CH}_{3}$ ), 3.83 (s, 3H, OMe), 4.24-4.32 (m, 2H, $\mathrm{OCH}_{2} \mathrm{CH}_{3}$ ), $6.22(\mathrm{~s}, 1 \mathrm{H}, \mathrm{CHCN}), 6.97-7.00\left(\mathrm{~m}, 1 \mathrm{H}, o-\mathrm{C}_{6} \mathrm{H}_{4} \mathrm{OMe}\right), 7.04-7.05$ (m, $1 \mathrm{H}, o-\mathrm{C}_{6} \mathrm{H}_{4} \mathrm{OMe}$ ), 7.09-7.11 (m, $\left.1 \mathrm{H} p-\mathrm{C}_{6} \mathrm{H}_{4} \mathrm{OMe}\right), 7.33-7.37$ $\left(\mathrm{m}, 1 \mathrm{H}, m-\mathrm{C}_{6} \mathrm{H}_{4} \mathrm{OMe}\right) \mathrm{ppm} .{ }^{13} \mathrm{C} \mathrm{NMR}\left(400 \mathrm{MHz}, \mathrm{CDCl}_{3}\right) \delta: 14.1$, 55.4, 65.6, 66.2, 113.0, 116.4, 116.5, 120.0, 130.4, 132.5, 153.4, 160.1 ppm. APCI: $m / z$ calculated for $\mathrm{C}_{12} \mathrm{H}_{14} \mathrm{NO}_{4}[\mathrm{M}+\mathrm{H}]^{+}=$ 236.0923, found: 236.0955 .

Cyano(cyclohexyl)methyl ethyl carbonate (7p). Prepared according to Method B (reaction time $12 \mathrm{~h}$ ). The residue was purified by column chromatography (hexanes/EtOAc $90: 10$ ) to obtain $180 \mathrm{mg}(85 \%)$ of a colorless oil. ${ }^{1} \mathrm{H}$ NMR $(400 \mathrm{MHz}$, $\left.\mathrm{CDCl}_{3}\right) \delta: 1.15-1.29(\mathrm{~m}, 5 \mathrm{H}), 1.33\left(\mathrm{t}, 3 \mathrm{H}, J=7.13 \mathrm{~Hz}, \mathrm{OCH}_{2} \mathrm{CH}_{3}\right)$, 1.68-1.93 (m, 6H), 4.21-4.31 (m, 2H OCH $\left.\mathrm{CH}_{3}\right), 5.03(\mathrm{~d}, J=$ $5.88 \mathrm{~Hz}, 1 \mathrm{H}, \mathrm{CHCN}) \mathrm{ppm} .{ }^{13} \mathrm{C} \mathrm{NMR}\left(400 \mathrm{MHz}, \mathrm{CDCl}_{3}\right) \delta: 14.2$, 25.3, 25.8, 28.1, 40.3, 65.5, 69.4. APCI: $m / z$ calculated for $\mathrm{C}_{11} \mathrm{H}_{18} \mathrm{NO}_{3}[\mathrm{M}+\mathrm{H}]^{+}=212.1287$, found: 212.1255 . 


\section{General procedure for conjugate additions}

$1 \mathrm{M}$ LiHMDS (THF) $(750 \mu \mathrm{L}, 0.75 \mathrm{mmol})$ was slowly added to a solution of cyanocarbonate $(0.5 \mathrm{mmol})$ in THF $(2.5 \mathrm{~mL})$ at $-78{ }^{\circ} \mathrm{C}$ under nitrogen atmosphere, and the resultant mixture stirred at this temperature for $15 \mathrm{~min}$. Then, the enone 0.75 mmol) was added dropwise, and after the addition was completed, the mixture was allowed to reach ambient temperature. When the starting material has been consumed (TLC), the reaction was quenched by the slow addition of sat $\mathrm{NH}_{4} \mathrm{Cl}(5$ $\mathrm{mL})$. The mixture was diluted with water and extracted with EtOAc $(3 \times 20 \mathrm{~mL})$; the organics were combined, dried (anh $\mathrm{Na}_{2} \mathrm{SO}_{4}$ ) and concentrated in vacuo. The residue was purified by column chromatography $\left(\mathrm{SiO}_{2}\right)$.

Methyl 6-benzoyl-2-hydroxycyclohex-1-ene-1-carboxylate (8a). Prepared following the general procedure (reaction time $2 \mathrm{~h})$. The residue was purified by column chromatography (hexanes/EtOAc 95 : 5) to obtain $103 \mathrm{mg}(79 \%)$ of a yellow oil. ${ }^{1} \mathrm{H}$ NMR (400 MHz, $\mathrm{CDCl}_{3}$ ) $\delta: 1.65-1.95\left(\mathrm{~m}, 4 \mathrm{H}, \mathrm{CH}_{2} \mathrm{CH}_{2} \mathrm{CH}_{2} \mathrm{CH}\right)$, 2.17-2.44 (m, 2H, $\mathrm{COHCH}_{2} \mathrm{CH}_{2}$ ), 3.55 (s, 3H, OMe), 4.48-4.50 (m, 1H, CH), 7.44-7.46 (m, 2H, m- $\left.\mathrm{C}_{6} \mathrm{H}_{5}\right), 7.47-7.48(\mathrm{~m}, 1 \mathrm{H}, p$ $\mathrm{C}_{6} \mathrm{H}_{5}$ ), 7.95-7.98 (m, 2H,o- $\mathrm{C}_{6} \mathrm{H}_{5}$ ), 12.34 (bs, $\left.1 \mathrm{H}, \mathrm{OH}\right) \mathrm{ppm} .{ }^{13} \mathrm{C}$ NMR (400 MHz, $\left.\mathrm{CDCl}_{3}\right) \delta: 18.5,26.1,28.9,41.0,51.5,97.2,128.3$, 128.7, 132.9, 133.7, 172.0, 174.5, 201.7 ppm. IR (cm $\left.{ }^{-1}\right): 3334$, 1738, 1714, 1673, 1333. APCI: $m / z$ calculated for $\mathrm{C}_{15} \mathrm{H}_{17} \mathrm{O}_{4}[\mathrm{M}+$ $\mathrm{H}]^{+}=261.1127$, found: 261.1064 .

Ethyl 6-benzoyl-2-hydroxycyclohex-1-ene-1-carboxylate (8b). Prepared following the general procedure (reaction time $2 \mathrm{~h}$ ). The residue was purified by column chromatography (hexanes/ EtOAc 95 : 5) to obtain $103 \mathrm{mg}$ (75\%) of a colorless oil. ${ }^{1} \mathrm{H}$ NMR $\left(400 \mathrm{MHz}, \mathrm{CDCl}_{3}\right) \delta: 0.97\left(\mathrm{t}, 3 \mathrm{H}, J=7.12 \mathrm{~Hz}, \mathrm{OCH}_{2} \mathrm{CH}_{3}\right), 1.67-$ $1.94\left(\mathrm{~m}, 4 \mathrm{H}, \mathrm{CH}_{2} \mathrm{CH}_{2} \mathrm{CH}_{2} \mathrm{CH}\right), 2.32-2.39\left(\mathrm{~m}, 2 \mathrm{H}, \mathrm{COHCH}_{2} \mathrm{CH}_{2}\right)$, 4.01-4.06 (m, 2H, $\left.\mathrm{OCH}_{2} \mathrm{CH}_{3}\right), 4.50-4.53(\mathrm{~m}, 1 \mathrm{H}, \mathrm{CH}), 7.46-7.49$ $\left(\mathrm{m}, 2 \mathrm{H}, m-\mathrm{C}_{6} \mathrm{H}_{5}\right), 7.55-7.57\left(\mathrm{~m}, 1 \mathrm{H}, p-\mathrm{C}_{6} \mathrm{H}_{5}\right), 7.96-8.01(\mathrm{~m}, 2 \mathrm{H}, o-$ $\mathrm{C}_{6} \mathrm{H}_{5}$ ), 12.37 (bs, $\left.1 \mathrm{H}, \mathrm{OH}\right) \mathrm{ppm} .{ }^{13} \mathrm{C}$ NMR (400 MHz, $\mathrm{CDCl}_{3}$ ) $\delta$ : 13.9, 18.8, 26.3, 29.0, 41.0, 60.5, 97.6, 128.4, 128.7, 132.9, 136.6, 171.7, 174.5, 202.1 ppm. IR ( $\left.\mathrm{cm}^{-1}\right): 3448,1740,1682,1651$, 1334. APCI: $m / z$ calculated for $\mathrm{C}_{16} \mathrm{H}_{19} \mathrm{O}_{4}[\mathrm{M}+\mathrm{H}]^{+}=275.1283$; found: 275.1242 .

tert-Butyl 6-benzoyl-2-hydroxycyclohex-1-ene-1-carboxylate (8c). Prepared following the general procedure (reaction time $5 \mathrm{~h})$. The residue was purified by column chromatography (hexanes/EtOAc $90: 10)$ to obtain $94 \mathrm{mg}(62 \%)$ of a colorless oil. ${ }^{1} \mathrm{H}$ NMR (400 MHz, $\mathrm{CDCl}_{3}$ ) $\delta: 1.21$ (s, 9H, Ot-Bu), 1.63-1.74 (m, $\left.4 \mathrm{H}, \mathrm{CH}_{2} \mathrm{CH}_{2} \mathrm{CH}_{2} \mathrm{CH}\right), 2.30-2.34\left(\mathrm{~m}, 2 \mathrm{H}, \mathrm{COHCH}_{2} \mathrm{CH}_{2}\right), 4.43-$ 4.47 (m, 1H, CH), 7.43-7.53 (m, 2H, $\left.p-\mathrm{C}_{6} \mathrm{H}_{5}\right), 7.49-7.55(\mathrm{~m}, 1 \mathrm{H}$, $\left.m-\mathrm{C}_{6} \mathrm{H}_{5}\right), 7.94-8.01\left(\mathrm{~m}, 2 \mathrm{H}, o-\mathrm{C}_{6} \mathrm{H}_{5}\right), 12.55$ (bs, $\left.1 \mathrm{H}, \mathrm{OH}\right) \mathrm{ppm} .{ }^{13} \mathrm{C}$ NMR (400 MHz, CDCl3) $\delta: 19.0,26.3,28.1,29.1,41.3,81.9,98.8$, 128.4, 128.7, 132.9, 133.6, 168.4, 173.8, $202.0 \mathrm{ppm}$. IR $\left(\mathrm{cm}^{-1}\right)$ : 3061, 1716, 1683, 1648, 1317. APCI: $m / z$ calculated for $\mathrm{C}_{14} \mathrm{H}_{13} \mathrm{O}_{3}$ $[\mathrm{M}+\mathrm{H}]^{+}=303.1596$; found: 303.1594 .

Methyl 6-(furan-2-carbonyl)-2-hydroxycyclohex-1-ene-1carboxylate (8d). Prepared following the general procedure (reaction time $2 \mathrm{~h}$ ). The residue was purified by column chromatography (hexanes/EtOAc $90: 10)$ to obtain $102 \mathrm{mg}(82 \%)$ of a light brown oil. ${ }^{1} \mathrm{H}$ NMR $\left(400 \mathrm{MHz}, \mathrm{CDCl}_{3}\right) \delta: 1.60-1.67(\mathrm{~m}, 2 \mathrm{H}$, $\mathrm{CH}_{2} \mathrm{CH}_{2} \mathrm{CH}_{2}$ ), 1.83-1.92 (m, 2H, $\left.\mathrm{CH}_{2} \mathrm{CH}_{2} \mathrm{CH}\right), 2.27-2.32(\mathrm{~m}, 2 \mathrm{H}$,
$\mathrm{COHCH}_{2} \mathrm{CH}_{2}$ ), 3.55 (s, 3H, OMe), 4.22-4.23 (m, 1H, CH), 6.51$6.53\left(\mathrm{~m}, 1 \mathrm{H}, 4-\mathrm{C}_{4} \mathrm{H}_{3} \mathrm{O}\right), 7.19-7.20\left(\mathrm{~m}, 1 \mathrm{H}, 3-\mathrm{C}_{4} \mathrm{H}_{3} \mathrm{O}\right), 7.57-7.59$ $\left(\mathrm{m}, 1 \mathrm{H}, 5-\mathrm{C}_{4} \mathrm{H}_{3} \mathrm{O}\right), 12.33$ (bs, $\left.1 \mathrm{H}, \mathrm{OH}\right) \mathrm{ppm} .{ }^{13} \mathrm{C}$ NMR $(400 \mathrm{MHz}$, $\left.\mathrm{CDCl}_{3}\right) \delta:$ 18.6, 26.4, 28.9, 41.8, 51.6, 96.5, 112.3, 117.3, 146.4, 147.1, 172.0, 174.9, 190.8 ppm. IR ( $\left.\mathrm{cm}^{-1}\right): 3321,1732,1715$, 1666, 1328. APCI: $m / z$ calculated for $\mathrm{C}_{13} \mathrm{H}_{15} \mathrm{O}_{5}[\mathrm{M}+\mathrm{H}]^{+}=$ 251.0919; found: 251.0824 .

tert-Butyl 2-(3-hydroxy-2-(methoxycarbonyl)cyclohex-2-ene-1carbonyl)-1H-pyrrole-1-carboxylate (8e). Prepared following the general procedure (reaction time $12 \mathrm{~h}$ ). The residue was purified by column chromatography (hexanes/EtOAc $70: 30$ ) to obtain $128 \mathrm{mg}(70 \%)$ of a light brown oil. ${ }^{1} \mathrm{H} \mathrm{NMR}\left(400 \mathrm{MHz}, \mathrm{CDCl}_{3}\right) \delta$ : $1.09\left(\mathrm{t}, 3 \mathrm{H}, J=7.13 \mathrm{~Hz}, \mathrm{OCH}_{2} \mathrm{CH}_{3}\right), 1.56(\mathrm{~s}, 9 \mathrm{H}, \mathrm{Ot}-\mathrm{Bu}), 1.80-1.93$ (m, 4H, $\left.\mathrm{CH}_{2} \mathrm{CH}_{2} \mathrm{CH}_{2} \mathrm{CH}\right), 2.32-2.37$ (m, 3H, $\mathrm{COHCH}_{2} \mathrm{CH}_{2}$ ), 3.93-4.15 (m, 3H, OCH $\left.\mathrm{CH}_{3}, \mathrm{CH}\right), 6.16-6.20\left(\mathrm{~m}, 1 \mathrm{H}, 4-\mathrm{C}_{4} \mathrm{H}_{3} \mathrm{~N}\right)$, 6.98-6.99 (m, 1H, 3- $\left.\mathrm{C}_{4} \mathrm{H}_{3} \mathrm{~N}\right), 7.37-7.38\left(\mathrm{~m}, 1 \mathrm{H}, 5-\mathrm{C}_{4} \mathrm{H}_{3} \mathrm{~N}\right), 12.42$ (bs, $1 \mathrm{H}, \mathrm{OH}$ ) ppm. ${ }^{13} \mathrm{C} \mathrm{NMR}\left(400 \mathrm{MHz}, \mathrm{CDCl}_{3}\right) \delta: 14.0,18.8,26.0$, 27.7, 29.1, 50.8, 60.6, 84.9, 97.1, 109.7, 121.4, 128.7, 133.3, 149.2, 169.2, 174.7, 192.0 ppm. IR ( $\left.\mathrm{cm}^{-1}\right): 3476,1744,1717,1673$, 1619, 1310. APCI: $m / z$ calculated for $\mathrm{C}_{19} \mathrm{H}_{25} \mathrm{NO}_{6}[\mathrm{M}+\mathrm{H}]^{+}=$ 364.1760; found: 364.1737.

Methyl 6-(thiophen-2-carbonyl)-2-hydroxycyclohex-1-ene-1carboxylate (8f). Prepared following the general procedure (reaction time $2 \mathrm{~h}$ ). The residue was purified by column chromatography (hexanes/EtOAc $95: 5)$ to obtain $97 \mathrm{mg}(69 \%)$ of a yellow oil. ${ }^{1} \mathrm{H}$ NMR $\left(400 \mathrm{MHz}, \mathrm{CDCl}_{3}\right) \delta: 0.93(\mathrm{t}, 3 \mathrm{H}, J=$ $7.15 \mathrm{~Hz}, \mathrm{OCH}_{2} \mathrm{CH}_{3}$ ), 1.84-1.9 (m, 4H, $\left.\mathrm{CH}_{2} \mathrm{CH}_{2} \mathrm{CH}_{2} \mathrm{CH}\right), 2.30-$ $2.34\left(\mathrm{~m}, 2 \mathrm{H}, \mathrm{COHCH}_{2} \mathrm{CH}_{2}\right), 3.96-4.02\left(\mathrm{~m}, 2 \mathrm{H}, \mathrm{OCH}_{2} \mathrm{CH}_{3}\right), 4.24-$ $4.27(\mathrm{~m}, 1 \mathrm{H}, \mathrm{CH}), 7.10-7.12\left(\mathrm{~m}, 1 \mathrm{H}, 4-\mathrm{C}_{4} \mathrm{H}_{3} \mathrm{~S}\right), 7.59-7.61(\mathrm{~m}, 1 \mathrm{H}$, 3- $\mathrm{C}_{4} \mathrm{H}_{3} \mathrm{~S}$ ), 7.62-7.77 (m, 1H, 5- $\mathrm{C}_{4} \mathrm{H}_{3} \mathrm{~S}$ ), 12.36 (bs, 1H, OH) ppm. ${ }^{13} \mathrm{C}$ NMR (400 MHz, $\mathrm{CDCl}_{3}$ ) $\delta: 13.7,19.0,26.9,29.0,43.0,60.5$, $97.4,128.1,131.7,133.4,143.3,171.6,174.7,195.0$ ppm. IR $\left(\mathrm{cm}^{-1}\right): 3091,1737,1715,1652,1333,1216$. APCI: $\mathrm{m} / z$ calculated for $\mathrm{C}_{14} \mathrm{H}_{17} \mathrm{O}_{4} \mathrm{~S}[\mathrm{M}+\mathrm{H}]^{+}=281.0848$; found: 281.0838 .

Methyl 6-(2-bromobenzoyl)-2-hydroxycyclohex-1-ene-1carboxylate (8g). Prepared following the general procedure (reaction time $1 \mathrm{~h}$ ). The residue was purified by column chromatography (hexanes/EtOAc $80: 20$ ) to obtain $143 \mathrm{mg}(84 \%)$ of a colorless oil. ${ }^{1} \mathrm{H}$ NMR $\left(400 \mathrm{MHz}, \mathrm{CDCl}_{3}\right) \delta: 1.68-1.77(\mathrm{~m}, 4 \mathrm{H}$, $\left.\mathrm{CH}_{2} \mathrm{CH}_{2} \mathrm{CH}_{2} \mathrm{CH}\right), 2.31-2.45\left(\mathrm{~m}, 2 \mathrm{H}, \mathrm{COHCH}_{2} \mathrm{CH}_{2}\right), 3.70(\mathrm{~s}, 3 \mathrm{H}$, OMe), $4.22-4.24$ (m, $1 \mathrm{H}, \mathrm{CH}), 7.26-7.31$ (m, $1 \mathrm{H}, \mathrm{m}_{-} \mathrm{C}_{6} \mathrm{H}_{4} \mathrm{Br}$ ), 7.36-7.40 (m, $\left.1 \mathrm{H}, m-\mathrm{C}_{6} \mathrm{H}_{4} \mathrm{Br}\right)$, 7.52-7.54 (m, 1H, $\left.p-\mathrm{C}_{6} \mathrm{H}_{4} \mathrm{Br}\right), 7.60-$ $7.64\left(\mathrm{~m}, 1 \mathrm{H}, o-\mathrm{C}_{6} \mathrm{H}_{4} \mathrm{Br}\right), 12.36$ (bs, $\left.1 \mathrm{H}, \mathrm{OH}\right) \mathrm{ppm} .{ }^{13} \mathrm{C} \mathrm{NMR}(400$ $\left.\mathrm{MHz}, \mathrm{CDCl}_{3}\right) \delta: 18.5,24.4,29.0,45.3,51.7,96.6,119.9,127.3$, 128.5, 131.6, 134.1, 141.2, 172.1, 174.9, $203.6 \mathrm{ppm}$. IR $\left(\mathrm{cm}^{-1}\right)$ : $3440,1742,1702,1655,1333,1087$. APCI: $m / z$ calculated for $\mathrm{C}_{15} \mathrm{H}_{16} \mathrm{BrO}_{4}[\mathrm{M}+\mathrm{H}]^{+}=339.0232$; found: 239.0232 .

Ethyl 6-(2-bromobenzoyl)-2-hydroxycyclohex-1-ene-1carboxylate (8h). Prepared following the general procedure (reaction time $5 \mathrm{~h}$ ). The residue was purified by column chromatography (hexanes/EtOAc $90: 10)$ to obtain $153 \mathrm{mg}(87 \%)$ of a colorless oil (mp $=92-94{ }^{\circ} \mathrm{C}$, EtOAc). ${ }^{1} \mathrm{H}$ NMR $(400 \mathrm{MHz}$, $\left.\mathrm{CDCl}_{3}\right) \delta: 1.20\left(\mathrm{t}, 3 \mathrm{H}, J=7.17 \mathrm{~Hz} \mathrm{OCH}_{2} \mathrm{CH}_{3}\right), 1.63-1.81(\mathrm{~m}, 4 \mathrm{H}$, $\left.\mathrm{CH}_{2} \mathrm{CH}_{2} \mathrm{CH}_{2} \mathrm{CH}\right)$ 2.32-2.44 (m, 2H, $\left.\mathrm{COHCH}_{2} \mathrm{CH}_{2}\right), 4.13-4.19(\mathrm{~m}$, $2 \mathrm{H}, 7.38)\left(\mathrm{m}, 1 \mathrm{H}, m-\mathrm{C}_{6} \mathrm{H}_{4} \mathrm{Br}\right), 7.54-7.56\left(\mathrm{~m}, 1 \mathrm{H}, p-\mathrm{C}_{6} \mathrm{H}_{4} \mathrm{Br}\right), 7.59-$ $7.76\left(\mathrm{~m}, 1 \mathrm{H}, o-\mathrm{C}_{6} \mathrm{H}_{4} \mathrm{Br}\right), 12.46$ (bs, $\left.1 \mathrm{H}, \mathrm{OH}\right) \mathrm{ppm} .{ }^{13} \mathrm{C} \mathrm{NMR}(400$ $\left.\mathrm{MHz}, \mathrm{CDCl}_{3}\right) \delta: 14.2,18.3,24.1,28.9,45.2,60.6,96.5,119.8$, 
127.1, 128.3, 131.5, 133.9, 141.1, 169.4, 174.8, 203.4 ppm. IR $\left(\mathrm{cm}^{-1}\right): 3064,1703,1651,1617,1304,1086$. APCI: $\mathrm{m} / z$ calculated for $\mathrm{C}_{16} \mathrm{H}_{18} \mathrm{BrO}_{3}[\mathrm{M}+\mathrm{H}]^{+}=353.0388$; found: 353.0352 .

Methyl

2-hydroxy-6-(2-nitrobenzoyl)cyclohex-1-ene-1carboxylate (8i). Prepared following the general procedure (reaction time $30 \mathrm{~min}$ ). The residue was purified by column chromatography (hexanes/EtOAc $90: 10$ ) to obtain $132 \mathrm{mg}$ (86\%) of a light-yellow oil. ${ }^{1} \mathrm{H}$ NMR $\left(400 \mathrm{MHz}^{\mathrm{CDCl}} 3\right.$ ) $\delta: 1.61-$ $1.71\left(\mathrm{~m}, 4 \mathrm{H}, \mathrm{CH}_{2} \mathrm{CH}_{2} \mathrm{CH}_{2} \mathrm{CH}\right), 2.26-2.30\left(\mathrm{~m}, 2 \mathrm{H}, \mathrm{COHCH}_{2} \mathrm{CH}_{2}\right.$ ), 3.63 (s, 3H, OMe), 4.16-4.17 (m, 1H, CH), 7.20-7.24 (m, 1H, m$\mathrm{C}_{6} \mathrm{H}_{4} \mathrm{NO}_{2}$ ), 7.30-7.33 (m, $1 \mathrm{H} m-\mathrm{C}_{6} \mathrm{H}_{4} \mathrm{NO}_{2}$ ), 7.46-7.48 (m, $1 \mathrm{H}, p$ $\left.\mathrm{C}_{6} \mathrm{H}_{4} \mathrm{NO}_{2}\right)$, $7.55-7.57\left(\mathrm{~m}, 1 \mathrm{H}, o-\mathrm{C}_{6} \mathrm{H}_{4} \mathrm{NO}_{2}\right), 12.30$ (bs, $1 \mathrm{H}$, $\mathrm{OH})$ ppm. ${ }^{13} \mathrm{C} \mathrm{NMR}\left(400 \mathrm{MHz}, \mathrm{CDCl}_{3}\right) \delta: 18.5,24.3,28.9,45.3$, 51.6, 96.6, 119.8, 127.3, 128.4, 131.6, 134.0, 141.1, 172.0, 174.9, 203.6 ppm. IR ( $\left.\mathrm{cm}^{-1}\right): 3001,1742,1702,1657,1440$. APCI: $\mathrm{m} / z$ calculated for $\mathrm{C}_{15} \mathrm{H}_{16} \mathrm{NO}_{6}\left[\mathrm{M}-\mathrm{CO}_{2} \mathrm{Me}\right]^{+}=247.0845$; found: 247.0862 .

Methyl 2-hydroxy-6-(3-methoxybenzoyl)cyclohex-1-ene-1carboxylate (8j). Prepared following the general procedure (reaction time $6 \mathrm{~h}$ ). The residue was purified by column chromatography (hexanes/EtOAc $90: 10)$ to obtain $77 \mathrm{mg}(50 \%)$ of a white solid. ${ }^{1} \mathrm{H}$ NMR $\left(400 \mathrm{MHz}, \mathrm{CDCl}_{3}\right) \delta: 0.98(\mathrm{t}, 3 \mathrm{H}, J=$ $\left.7.13 \mathrm{~Hz}, \mathrm{OCH}_{2} \mathrm{CH}_{3}\right), 1.62-1.79\left(\mathrm{~m}, 4 \mathrm{H}, \mathrm{CH}_{2} \mathrm{CH}_{2} \mathrm{CH}_{2} \mathrm{CH}\right), 2.31-$ $2.37\left(\mathrm{~m}, 2 \mathrm{H}, \mathrm{COHCH}_{2} \mathrm{CH}_{2}\right), 3.85(\mathrm{~s}, 3 \mathrm{H}, \mathrm{OMe}), 4.01-4.02(\mathrm{~m}, 2 \mathrm{H}$, $\left.\mathrm{OCH}_{2} \mathrm{CH}_{3}\right), 4.46-4.48(\mathrm{~m}, 1 \mathrm{H}, \mathrm{CH}), 7.08-7.11\left(\mathrm{~m}, 1 \mathrm{H}, o-\mathrm{C}_{6} \mathrm{H}_{4}\right)$, 7.35-7.39 (m, $\left.1 \mathrm{H}, o-\mathrm{C}_{6} \mathrm{H}_{4}\right), 7.49-7.50\left(\mathrm{~m}, 1 \mathrm{H}, m-\mathrm{C}_{6} \mathrm{H}_{4}\right), 7.56-7.58$ $\left(\mathrm{m}, 1 \mathrm{H}, p-\mathrm{C}_{6} \mathrm{H}_{4}\right), 12.42$ (bs, $\left.1 \mathrm{H}, \mathrm{OH}\right) \mathrm{ppm} .{ }^{13} \mathrm{C}$ NMR $(400 \mathrm{MHz}$, $\left.\mathrm{CDCl}_{3}\right) \delta: 13.8,18.6,26.1,28.8,41.0,55.4,60.4,97.5,112.9$, 119.0, 120.7, 129.5, 137.8, 159.8, 171.6, 174.3, 201.8 ppm. IR $\left(\mathrm{cm}^{-1}\right): 3074,1738,1716,1682,1329$. APCI: $\mathrm{m} / \mathrm{z}$ calculated for $\mathrm{C}_{17} \mathrm{H}_{21} \mathrm{O}_{5}[\mathrm{M}+\mathrm{H}]^{+}=305.1389$; found: 305.1392 .

Ethyl 2-hydroxy-6-picolinoylcyclohex-1-ene-1-carboxylate (8k). Prepared following the general procedure (reaction time $10 \mathrm{~h}$ ). The residue was purified by column chromatography (hexanes/EtOAc $70: 30)$ to obtain $84 \mathrm{mg}(61 \%)$ of a brown oil. ${ }^{1} \mathrm{H}$ NMR (400 MHz, CDCl3) $\delta: 0.88\left(\mathrm{t}, 3 \mathrm{H}, J=7.17 \mathrm{~Hz}, \mathrm{OCH}_{2} \mathrm{CH}_{3}\right.$ ), 1.68-1.77 (m, 4H, $\left.\mathrm{CH}_{2} \mathrm{CH}_{2} \mathrm{CH}_{2} \mathrm{CH}\right), 2.33-2.36(\mathrm{~m}, 2 \mathrm{H}$, $\left.\mathrm{COHCH}_{2} \mathrm{CH}_{2}\right), 3.96-4.01\left(\mathrm{~m}, 2 \mathrm{H}, \mathrm{OCH}_{2} \mathrm{CH}_{3}\right), 5.12-5.15(\mathrm{~m}, 1 \mathrm{H}$, $\mathrm{CH}), 7.47-7.49\left(\mathrm{~m}, 1 \mathrm{H}, 5-\mathrm{C}_{5} \mathrm{H}_{4} \mathrm{~N}\right), 7.82-7.84\left(\mathrm{~m}, 1 \mathrm{H}, 3-\mathrm{C}_{5} \mathrm{H}_{4} \mathrm{~N}\right)$, 8.05-8.06 (m, $\left.1 \mathrm{H}, 4-\mathrm{C}_{5} \mathrm{H}_{4} \mathrm{~N}\right), 8.69-8.71\left(\mathrm{~m}, 1 \mathrm{H}, 6-\mathrm{C}_{5} \mathrm{H}_{4} \mathrm{~N}\right), 12.35$ (bs, $1 \mathrm{H}, \mathrm{OH}) \mathrm{ppm} .{ }^{13} \mathrm{C} \mathrm{NMR}\left(400 \mathrm{MHz}, \mathrm{CDCl}_{3}\right) \delta: 13.6,19.0,25.9$, 29.0, 39.2, 60.2, 97.7, 122.3, 126.9, 136.9, 148.8, 152.7, 169.2, 174.2, 202.8 ppm. IR ( $\left.\mathrm{cm}^{-1}\right): 3054.9,1739,1695,1652,1583$, 1347. APCI: $m / z$ calculated for $\mathrm{C}_{15} \mathrm{H}_{18} \mathrm{NO}_{4}[\mathrm{M}+\mathrm{H}]^{+}=276.1236$, found: 276.1200 .

Ethyl 2-hydroxy-6-nicotinoylcyclohex-1-ene-1-carboxylate (81). Prepared following the general procedure (reaction time $1.5 \mathrm{~h}$ ). The residue was purified by column chromatography (hexanes/EtOAc $70: 30$ ) to obtain $105 \mathrm{mg}$ (76\%) of a light-yellow oil. ${ }^{1} \mathrm{H}$ NMR (400 MHz, DMSO) $\delta: 0.91(\mathrm{t}, 3 \mathrm{H}, J=7.12 \mathrm{~Hz}$, $\mathrm{OCH}_{2} \mathrm{CH}_{3}$ ), 1.55-1.72 (m, 4H, $\left.\mathrm{CH}_{2} \mathrm{CH}_{2} \mathrm{CH}_{2} \mathrm{CH}\right), 2.25-2.43(\mathrm{~m}$, $\left.2 \mathrm{H}, \mathrm{COHCH}_{2} \mathrm{CH}_{2}\right), 3.97-4.13\left(\mathrm{~m}, 2 \mathrm{H}, \mathrm{OCH}_{2} \mathrm{CH}_{3}\right), 4.41-4.43(\mathrm{~m}$, $1 \mathrm{H}, \mathrm{CH}), 7.22-7.39\left(\mathrm{~m}, 1 \mathrm{H}, 5-\mathrm{C}_{5} \mathrm{H}_{4} \mathrm{~N}\right), 8.19-8.21(\mathrm{~m}, 1 \mathrm{H}, 4-$ $\left.\mathrm{C}_{5} \mathrm{H}_{4} \mathrm{~N}\right), 8.72-8.73\left(\mathrm{~m}, 1 \mathrm{H}, 6-\mathrm{C}_{5} \mathrm{H}_{4} \mathrm{~N}\right), 9.12-9.16(\mathrm{~m}, 1 \mathrm{H}, 2-$ $\mathrm{C}_{5} \mathrm{H}_{4} \mathrm{~N}$ ), 12.29 (bs, $1 \mathrm{H}, \mathrm{OH}$ ) ppm. ${ }^{13} \mathrm{C}$ NMR (400 MHz, DMSO) $\delta$ : 14.0, 18.7, 25.9, 28.8, 48.4, 60.7, 97.6, 124.5, 131.5, 136.2, 149.7, 153.9, 171.5, 174.5, 201.7 ppm. IR $\left(\mathrm{cm}^{-1}\right): 3048,1737,1716$,
1784, 1584, 1331. APCI: $m / z$ calculated for $\mathrm{C}_{15} \mathrm{H}_{18} \mathrm{NO}_{4}[\mathrm{M}+\mathrm{H}]^{+}=$ 276.1236; found: 276.1204 .

Methyl 2-hydroxy-6-(4-methoxybenzoyl)cyclohex-1-ene-1carboxylate (8m). Prepared following the general procedure (reaction time $12 \mathrm{~h}$ ). The residue was purified by column chromatography (hexanes/EtOAc $90: 10)$ to obtain $77 \mathrm{mg}(53 \%)$ of a white solid. ${ }^{1} \mathrm{H}$ NMR (400 MHz, $\left.\mathrm{CDCl}_{3}\right) \delta: 1.61-1.93(\mathrm{~m}, 4 \mathrm{H}$, $\left.\mathrm{CH}_{2} \mathrm{CH}_{2} \mathrm{CH}_{2} \mathrm{CH}\right), 2.28-2.40\left(\mathrm{~m}, 2 \mathrm{H}, \mathrm{COHCH}_{2} \mathrm{CH}_{2}\right), 3.57(\mathrm{~s}, 3 \mathrm{H}$, $\mathrm{CO}_{2} \mathrm{Me}$ ), 3.88 (s, 3H, OMe), 4.45-4.48 (m, 1H, CH), 6.93-6.98 (m, $2 \mathrm{H}, m-\mathrm{C}_{6} \mathrm{H}_{4} \mathrm{OMe}$ ), $7.94-8.00\left(\mathrm{~m}, 2 \mathrm{H}, o-\mathrm{C}_{6} \mathrm{H}_{4} \mathrm{OMe}\right.$ ), 12.35 (bs, $1 \mathrm{H}$, $\mathrm{OH}) \mathrm{ppm} . \mathrm{RMN}{ }^{13} \mathrm{C} \mathrm{NMR}\left(400 \mathrm{MHz}, \mathrm{CDCl}_{3}\right) \delta$ : 18.7, 26.6, 29.0, $40.7,48.3,58.7,97.5,113.9,130.8,163.5,170.0,174.6$, 198.3 ppm. IR ( $\left.\mathrm{cm}^{-1}\right): 3328,1742,1714,1662,1334$. APCI: $m / z$ calculated for $\mathrm{C}_{16} \mathrm{H}_{19} \mathrm{NO}_{5}[\mathrm{M}+\mathrm{H}]^{+}=291.1232$; found: 291.1251 .

Methyl 6-(6-bromobenzo[d][1,3]dioxole-5-carbonyl)-2hydroxycyclohex-1-ene-1-carboxylate (8n). Prepared following the general procedure (reaction time $3 \mathrm{~h}$ ). The residue was purified by column chromatography (hexanes/EtOAc $80: 20$ ) to obtain $150 \mathrm{mg}$ (78\%) of a white solid $\left(\mathrm{mp}=92-94{ }^{\circ} \mathrm{C}\right.$ AcOEt ${ }^{1} \mathrm{H}$ NMR (400 MHz, $\left.\mathrm{CDCl}_{3}\right) \delta: 1.66-1.76\left(\mathrm{~m}, 4 \mathrm{H}, \mathrm{CH}_{2} \mathrm{CH}_{2} \mathrm{CH}_{2} \mathrm{CH}\right)$, 2.02-2.35 (m, 2H, $\mathrm{COHCH}_{2} \mathrm{CH}_{2}$ ), 3.69 (s, 3H, OMe), 4.15-4.17 $(\mathrm{m}, 1 \mathrm{H}, \mathrm{CH}), 6.03\left(\mathrm{~s}, 2 \mathrm{H}, \mathrm{OCH}_{2} \mathrm{O}\right), 7.03\left(\mathrm{~s}, 1 \mathrm{H}, m-\mathrm{C}_{6} \mathrm{H}_{2} \mathrm{Br}\right), 7.05$ $\left(\mathrm{s}, 1 \mathrm{H}, o-\mathrm{C}_{6} \mathrm{H}_{2} \mathrm{Br}\right), 12.32$ (bs, $\left.1 \mathrm{H}, \mathrm{OH}\right) \mathrm{ppm} .{ }^{13} \mathrm{C} \mathrm{NMR}(400 \mathrm{MHz}$, $\left.\mathrm{CDCl}_{3}\right) \delta:$ 18.5, 24.6, 29.0, 45.0, 51.7, 96.7, 102.5, 108.5, 112.0, 114.2, 134.0, 147.3, 150.0, 172.0, 174.9, 202.5 ppm. IR (cm $\left.{ }^{-1}\right)$ : 3384, 1716, 1699, 1662, 1328, 1037. APCI: $\mathrm{m} / \mathrm{z}$ calculated for $\mathrm{C}_{16} \mathrm{H}_{16} \mathrm{BrO}_{6}[\mathrm{M}+\mathrm{H}]^{+}=383.0130$; found: 383.0155 .

tert-Butyl 2-(3-hydroxy-2-(methoxycarbonyl)cyclohex-2-ene-1carbonyl)-1H-indole-1-carboxylate (80). Prepared following the general procedure (reaction time $6 \mathrm{~h}$ ). The residue was purified by column chromatography (hexanes/EtOAc $80: 20$ ) to obtain $123 \mathrm{mg}(60 \%)$ of a brown oil. ${ }^{1} \mathrm{H}$ NMR $\left(400 \mathrm{MHz}, \mathrm{CDCl}_{3}\right) \delta: 1.05$ $\left(\mathrm{t}, 3 \mathrm{H}, J=7.13 \mathrm{~Hz}, \mathrm{OCH}_{2} \mathrm{CH}_{3}\right), 1.60(\mathrm{~s}, 9 \mathrm{H}, \mathrm{Ot}-\mathrm{Bu}), 1.89-1.99(\mathrm{~m}$, $\left.4 \mathrm{H}, \mathrm{CH}_{2} \mathrm{CH}_{2} \mathrm{CH}_{2} \mathrm{CH}\right), 2.34-2.37\left(\mathrm{~m}, 2 \mathrm{H}, \mathrm{COHCH}_{2} \mathrm{CH}_{2}\right), 3.99-$ $4.14\left(\mathrm{~m}, 2 \mathrm{H}, \mathrm{OCH}_{2} \mathrm{CH}_{3}\right), 4.28-4.30(\mathrm{~m}, 1 \mathrm{H}, \mathrm{CH}), 7.23-7.27(\mathrm{~m}$, $\left.1 \mathrm{H}, 5-\mathrm{C}_{8} \mathrm{H}_{5} \mathrm{~N}\right), 7.30\left(\mathrm{~s}, 1 \mathrm{H}, 3-\mathrm{C}_{8} \mathrm{H}_{5} \mathrm{~N}\right) 7.37-7.44\left(\mathrm{~m}, 1 \mathrm{H}, 6-\mathrm{C}_{8} \mathrm{H}_{5} \mathrm{~N}\right)$, 7.62-7.65 (m, 1H, 4- $\left.\mathrm{C}_{8} \mathrm{H}_{5} \mathrm{~N}\right), 7.98-8.01\left(\mathrm{~m}, 1 \mathrm{H}, 7-\mathrm{C}_{8} \mathrm{H}_{5} \mathrm{~N}\right), 12.45$ (bs, $1 \mathrm{H}, \mathrm{OH}) \mathrm{ppm} .{ }^{13} \mathrm{C} \mathrm{NMR}\left(400 \mathrm{MHz}, \mathrm{CDCl}_{3}\right) \delta: 14.0,18.7,25.8$, 27.9, 29.0, 43.4, 60.5, 84.5, 96.7, 114.5, 115.7, 117.0, 122.5, 123.1, 127.3, 137.5, 138.9, 149.6, 169.2, 174.8, 193.5 ppm. IR $\left(\mathrm{cm}^{-1}\right)$ : $3053,1736,1679,1652,1613,1321$. APCI: $m / z$ calculated for $\mathrm{C}_{15} \mathrm{H}_{18} \mathrm{NO}_{4}[\mathrm{M}+\mathrm{H}]^{+}=414.1917$; found: 414.1865 .

Methyl 2-benzoyl-5-oxocyclopentane-1-carboxylate (9a). Prepared following the general procedure (reaction time $5 \mathrm{~h}$ ). The residue was purified by column chromatography (hexanes/ EtOAc $70: 30)$ to obtain $99 \mathrm{mg}(80 \%)$ of a translucent yellow oil. ${ }^{1} \mathrm{H}$ NMR (400 MHz, $\mathrm{CDCl}_{3}$ ) $\delta$ : 1.94-1.99 (m, $\left.1 \mathrm{H}, \mathrm{CH}_{2} \mathrm{CH}_{2} \mathrm{CH}\right)$, 2.44-2.58 (m, 3H, $\mathrm{COCH}_{2} \mathrm{CH}_{2} \mathrm{CH}$ ), 3.75 (s, 3H, OMe), 3.89-3.92 (d, $1 \mathrm{H}, J=9.39 \mathrm{~Hz}, \mathrm{COCHCO}), 4.50-5.57$ (m, $1 \mathrm{H}, \mathrm{CH}_{2} \mathrm{CHCO}$ ), 7.49-7.60 (m, 2H, $\left.m-\mathrm{C}_{6} \mathrm{H}_{5}\right), 7.61-7.62\left(\mathrm{~m}, 1 \mathrm{H}, p-\mathrm{C}_{6} \mathrm{H}_{5}\right), 8.02-8.05$ $\left(\mathrm{m}, 2 \mathrm{H}, o-\mathrm{C}_{6} \mathrm{H}_{5}\right) \mathrm{ppm} .{ }^{13} \mathrm{C} \mathrm{NMR}\left(400 \mathrm{MHz}, \mathrm{CDCl}_{3}\right) \delta: 25.9,37.9$, 47.2, 53.0, 57.1, 128.8, 129.1, 134.0, 135.4, 168.6, 199.0, 209.1 ppm. IR $\left(\mathrm{cm}^{-1}\right): 3053,1748,1724,1678,1321$. APCI: $\mathrm{m} / \mathrm{z}$ calculated for $\mathrm{C}_{13} \mathrm{H}_{11} \mathrm{O}_{3}[\mathrm{M}+\mathrm{H}]^{+}=247.0970$; found: 247.1008 .

Methyl 2-benzoyl-7-oxocycloheptane-1-carboxylate (9b). Prepared following the general procedure (reaction time $5 \mathrm{~h}$ ). The residue was purified by column chromatography (hexanes/ 
EtOAc $80: 20)$ to obtain $68 \mathrm{mg}(50 \%)$ of a pale-yellow oil. ${ }^{1} \mathrm{H}$ NMR (400 MHz, $\mathrm{CDCl}_{3}$ ) $\delta: 1.49-1.62\left(\mathrm{~m}, 2 \mathrm{H}, \mathrm{CH}_{2} \mathrm{CH}_{2} \mathrm{CH}_{2} \mathrm{CH}\right)$, 1.95-2.70 (m, 4H, $\left.\mathrm{CH}_{2} \mathrm{CH}_{2} \mathrm{CH}_{2} \mathrm{CH}\right), 2.63-2.73\left(\mathrm{~m}, 2 \mathrm{H}, \mathrm{COCH}_{2}-\right.$ $\mathrm{CH}_{2}$ ), 3.64 (s, 3H, OMe), 4.01-4.03 (m, 1H, COCHCO), 4.39-4.41 (d, $\left.1 \mathrm{H}, J=10.18 \mathrm{~Hz}, \mathrm{CH}_{2} \mathrm{CHCO}\right), 7.45-7.49\left(\mathrm{~m}, 2 \mathrm{H}, m-\mathrm{C}_{6} \mathrm{H}_{5}\right)$, 7.56-7.57 (m, 1H, $\left.p-\mathrm{C}_{6} \mathrm{H}_{5}\right)$, 7.95-7.97 (m, 2H, o- $\left.\mathrm{C}_{6} \mathrm{H}_{5}\right) \mathrm{ppm} .{ }^{13} \mathrm{C}$ NMR (400 MHz, $\mathrm{CDCl}_{3}$ ) $\delta: 24.1,26.9,31.7,43.2,45.5$, 52.6, 59.9, 128.6, 128.9, 133.5, 135.5, 170.0, 201.1, $207.6 \mathrm{ppm}$. IR $\left(\mathrm{cm}^{-1}\right)$ : 3061, 1742, 1702, 1679, 1322. APCI: $m / z$ calculated for $\mathrm{C}_{16} \mathrm{H}_{19} \mathrm{O}_{4}$ $[\mathrm{M}+\mathrm{H}]^{+}=275.1283$; found: 275.1294 .

Methyl 6-benzoyl-2-hydroxy-6-methylcyclohex-1-ene-1carboxylate (9c). Prepared following the general procedure (reaction time $24 \mathrm{~h}$ ). The residue was purified by column chromatography (hexanes/EtOAc $80: 20)$ to obtain $52 \mathrm{mg}(38 \%)$ of a yellow oil. ${ }^{1} \mathrm{H}$ NMR $\left(400 \mathrm{MHz}, \mathrm{CDCl}_{3}\right) \delta: 1.51\left(\mathrm{~s}, 3 \mathrm{H}, \mathrm{OCH}_{3}\right)$, 1.70-1.74 (m, 1H, $\left.\mathrm{CH}_{2} \mathrm{CH}_{2} \mathrm{C}\right), 1.90-1.94\left(\mathrm{~m}, 2 \mathrm{H}, \mathrm{CH}_{2} \mathrm{CH}_{2} \mathrm{CH}_{2}\right.$ ), 2.12-2.18 (m, 1H, $\mathrm{CH}_{2} \mathrm{CH}_{2} \mathrm{C}$ ), 2.48-2.54 (m, 2H, COHCH $\mathrm{CH}_{2}$ ), 3.39 (s, 3H, OMe), 7.32-7.36 (m, $\left.2 \mathrm{H}, m-\mathrm{C}_{6} \mathrm{H}_{5}\right), 7.40-7.45(\mathrm{~m}, 1 \mathrm{H}$, $\left.p-\mathrm{C}_{6} \mathrm{H}_{5}\right), 7.81-7.83\left(\mathrm{~m}, 2 \mathrm{H}, o-\mathrm{C}_{6} \mathrm{H}_{5}\right), 12.61(\mathrm{bs}, 1 \mathrm{H}, \mathrm{OH}) \mathrm{ppm} .{ }^{13} \mathrm{C}$ NMR (400 MHz, $\mathrm{CDCl}_{3}$ ) $\delta: 18.1,24.9,29.6,34.8,48.2,51.1,104.7$, $128.3(\times 2), 131.7,136.7,172.1,172.8,203.9$ ppm. 3056, 1717, 1678, 1650, 1308. APCI: $m / z$ calculated for $\mathrm{C}_{15} \mathrm{H}_{15} \mathrm{O}_{3}[\mathrm{M}+\mathrm{H}]^{+}=$ 275.1283; found: 275.1240 .

Ethyl 4-oxo-1-phenyl-3,4,5,6,7,7a-hexahydro-3aH-indene-3acarboxylate (10). DBU (109 $\mu \mathrm{L}, 0.73 \mathrm{mmol})$ was added to a solution of $30(100 \mathrm{mg}, 0.37 \mathrm{mmol})$ in acetonitrile $(3.7 \mathrm{~mL})$ at $0{ }^{\circ} \mathrm{C}$ under nitrogen atmosphere. After stirring the mixture at this temperature for $15 \mathrm{~min}$, triphenylvinylphosphonium bromide (202 mg, $546 \mathrm{mmol}$ ) was added portion wise. After the addition was completed, the mixture was stirring for $12 \mathrm{~h}$ at room temperature. The reaction was quenched by the addition of $\mathrm{H}_{2} \mathrm{O}(10 \mathrm{~mL})$ and extracted with EtOAc $(3 \times 20 \mathrm{~mL})$; the organics were combined, dried $\left(\mathrm{anh} \mathrm{Na}_{2} \mathrm{SO}_{4}\right)$ and concentrated in vacuo. The residue was purified by column chromatography $\left(\mathrm{SiO}_{2}\right)$ (hexanes/EtOAc $\left.70: 30\right)$ to obtain $40 \mathrm{mg}(39 \%)$ of translucent yellow oil. ${ }^{1} \mathrm{H}$ NMR $\left(400 \mathrm{MHz}, \mathrm{CDCl}_{3}\right) \delta: 1.25(\mathrm{t}, 3 \mathrm{H}, J=$ $\left.3.17 \mathrm{~Hz}, \mathrm{OCH}_{2} \mathrm{CH}_{3}\right), 1.56-1.78\left(\mathrm{~m}, 4 \mathrm{H}, \mathrm{CH}_{2} \mathrm{CH}_{2} \mathrm{CH}_{2} \mathrm{CH}\right), 2.11-$ $2.14\left(\mathrm{~m}, 1 \mathrm{H}, \mathrm{COHCH}_{2} \mathrm{CH}_{2}\right), 2.46-2.50\left(\mathrm{~m}, 1 \mathrm{H}, \mathrm{COHCH}_{2} \mathrm{CH}_{2}\right)$, 2.52-2.54 (m, $\left.1 \mathrm{H}, \mathrm{CCH}_{2} \mathrm{CH}\right), 3.06-3.07\left(\mathrm{~m}, 1 \mathrm{H}, \mathrm{CCH}_{2} \mathrm{CH}\right)$, $3.984 .00(\mathrm{~m}, 1 \mathrm{H}, \mathrm{CH}), 4.02-4.22\left(\mathrm{~m}, 2 \mathrm{H}, \mathrm{OCH}_{2} \mathrm{CH}_{3}\right), 5.93-5.95$ $\left(\mathrm{m}, 1 \mathrm{H}, \mathrm{CCHCH}_{2}\right), 7.31-7.35\left(\mathrm{~m}, 5 \mathrm{H}, \mathrm{C}_{6} \mathrm{H}_{5}\right) \mathrm{ppm} .{ }^{13} \mathrm{C}$ NMR (400 $\left.\mathrm{MHz} \mathrm{CDCl}_{3}\right) \delta: 14.1,21.5,26.3,38.3,39.3,51.5,61.7,65.8,125.0$, 126.3, 127.4, 128.4, 135.3, 144.4, 172.1, 208.7. IR ( $\left.\mathrm{cm}^{-1}\right): 2945$, 1709, 1676, 1315. APCI: $m / z$ calculated for $\mathrm{C}_{18} \mathrm{H}_{21} \mathrm{O}_{3}[\mathrm{M}+\mathrm{H}]^{+}=$ 285.1491; found: 285.1467.

2-Acetyl-1-phenyl-3,3a,5,6,7,7a-hexahydro-4H-inden-4-one (11). DBU ( $82 \mu \mathrm{L}, 0.55 \mathrm{mmol})$ was added to a solution of 30 $(100 \mathrm{mg}, 0.37 \mathrm{mmol})$ in acetonitrile $(1.2 \mathrm{~mL})$ at $0{ }^{\circ} \mathrm{C}$ under nitrogen atmosphere. After stirring the mixture at this temperature for $15 \mathrm{~min}$, methyl vinyl ketone ( $35 \mu \mathrm{L}, 401 \mathrm{mmol})$ was added dropwise. After the addition was completed, the mixture was stirring for $12 \mathrm{~h}$ at $80{ }^{\circ} \mathrm{C}$. The reaction was quenched by the addition of $\mathrm{H}_{2} \mathrm{O}(5 \mathrm{~mL})$. The mixture was diluted with water and extracted with EtOAc $(3 \times 20 \mathrm{~mL})$; the organics were combined, dried $\left(\right.$ anh $\left.\mathrm{Na}_{2} \mathrm{SO}_{4}\right)$ and concentrated in vacuo. The residue was purified by column chromatography $\left(\mathrm{SiO}_{2}\right)$ (hexanes/EtOAc $\left.80: 20\right)$ to obtain $82 \mathrm{mg}(88 \%)$ of translucent oil. ${ }^{1} \mathrm{H}$ NMR (400 MHz, $\left.\mathrm{CDCl}_{3}\right) \delta: 1.40-1.44(\mathrm{~m}, 1 \mathrm{H}$, $\mathrm{CH}_{2} \mathrm{CH}_{2} \mathrm{CH}_{2}$ ), 1.62-1.74 (m, $\left.1 \mathrm{H}, \mathrm{CH}_{2} \mathrm{CH}_{2} \mathrm{CH}_{2}\right), 1.75-1.77(\mathrm{~m}$, $\left.2 \mathrm{H}, \mathrm{CH}_{2} \mathrm{CH}_{2} \mathrm{CH}\right), 1.81$ (s, 3H, $\left.\mathrm{COCH}_{3}\right), 2.36-2.39(\mathrm{~m}, 2 \mathrm{H}$, $\left.\mathrm{COCH}_{2} \mathrm{CH}_{2}\right), 2.84-2.85\left(\mathrm{~m}, 1 \mathrm{H}, \mathrm{CHCH}_{2} \mathrm{C}\right), 2.97-2.99(\mathrm{~m}, 1 \mathrm{H}$, $\mathrm{CHCH}_{2} \mathrm{C}$ ), 3.12-3.15 (m, 1H, CH), 3.48-3.50 (m, 1H, CH), 7.12$7.15\left(\mathrm{~m}, 2 \mathrm{H}, m-\mathrm{C}_{6} \mathrm{H}_{5}\right), 7.32-7.37\left(\mathrm{~m}, 3 \mathrm{H}, o, p-\mathrm{C}_{6} \mathrm{H}_{5}\right) \mathrm{ppm} .{ }^{13} \mathrm{C}$ NMR (400 MHz, $\mathrm{CDCl}_{3}$ ) $\delta: 22.4,26.2,29.8,35.3,39.4,48.7,53.7$, 127.8, 128.4, 128.6, 136.2, 138.4, 155.1, 198.8, 212.4 ppm. IR $\left(\mathrm{cm}^{-1}\right): 1348,1682,1704,1720,2939$. APCI: $\mathrm{m} / \mathrm{z}$ calculated for $\mathrm{C}_{17} \mathrm{H}_{19} \mathrm{O}_{2}[\mathrm{M}+\mathrm{H}]^{+}=255.1385$; found: 255.1379.

8-Phenyl-2,3,3a,3b,5,6,7,7a-octahydrocyclopenta[a]indene1,4-dione (12). DBU ( $82 \mu \mathrm{L}, 0.55 \mathrm{mmol})$ was added to a solution of $30(100 \mathrm{mg}, 0.37 \mathrm{mmol})$ in acetonitrile $(1.2 \mathrm{~mL})$ at $0{ }^{\circ} \mathrm{C}$ under nitrogen atmosphere. After stirring the mixture at this temperature for $15 \mathrm{~min}, 2$-cyclopenten-1-one (34 $\mu \mathrm{L}, 401 \mathrm{mmol})$ was added dropwise. After the addition was completed, the mixture was stirring for $24 \mathrm{~h}$ at room temperature. The reaction was quenched by the addition of $\mathrm{H}_{2} \mathrm{O}(5 \mathrm{~mL})$. The mixture was diluted with water and extracted with EtOAc $(3 \times 20 \mathrm{~mL})$; the organics were combined, dried ( $\left.\mathrm{anh} \mathrm{Na}_{2} \mathrm{SO}_{4}\right)$ and concentrated in vacuo. The residue was purified by column chromatography $\left(\mathrm{SiO}_{2}\right)$ (hexanes/EtOAc $\left.80: 20\right)$ to obtain $31 \mathrm{mg}$ (32\%) of translucent oil. ${ }^{1} \mathrm{H}$ NMR (400 MHz, $\mathrm{CDCl}_{3}$ ) $\delta: 1.51-1.61(\mathrm{~m}, 1 \mathrm{H}), 1.71-$ $1.80(\mathrm{~m}, 2 \mathrm{H}), 2.02-2.10(\mathrm{~m}, 2 \mathrm{H}), 2.24-2.37$ (m, 1H), 2.47-2.50 (m, 2H), 2.61-2.65 (m, 2H), 2.84-2.89 (m, 1H), 3.63-3.70 (m, 1H, $\mathrm{CH})$, 3.88-3.94 (m, 1H, CH), 7.39-7.43 (m, 2H, $\left.m-\mathrm{C}_{6} \mathrm{H}_{5}\right)$, 7.99$8.02\left(\mathrm{~m}, 3 \mathrm{H}, o, p-\mathrm{C}_{6} \mathrm{H}_{5}\right) \mathrm{ppm} .{ }^{13} \mathrm{C} \mathrm{NMR}\left(400 \mathrm{MHz}, \mathrm{CDCl}_{3}\right) \delta: 24.0$, 27.8, 28.9, 39.1, 44.7, 53.3, 54.9, 59.3, 128.5, 129.5, 130.5, 132.1, 137.6, 151.2, 200.5, 212.3 ppm. IR $\left(\mathrm{cm}^{-1}\right): 1335,1590,1686$, 2951. APCI: $m / z$ calculated for $\mathrm{C}_{17} \mathrm{H}_{19} \mathrm{O}_{2}[\mathrm{M}+\mathrm{H}]^{+}=267.1385$; found: 267.1349 .

\section{Conclusions}

We utilized a cascade process to obtain tricarbonyl compounds by adding anions of cyanocarbonates derived from aromatic aldehydes onto 5, 6, and 7-membered cycloalkenones. During the process, we exploited the dual role of cyanocarbonates: as "latent" acylcarbanions and as acylating reagents. The synthetic potential of the cascade products obtained was successfully demonstrated by forming bicyclic and tricyclic systems through intramolecular condensation reactions. These results suggest that tricarbonyl compounds can be used as scaffolds to generate structural diversity. The investigation of further transformations is currently underway and will be published in due course.

\section{Conflicts of interest}

There are no conflicts to declare.

\section{Acknowledgements}

The authors thank Facultad de Química, UNAM for financial support (Grant PAIP 50009062). We thank Professor D. E. Ward for revising the manuscript, and for helpful suggestions. We also thank Rosa Isela del Villar, Nayeli López, Georgina Duarte, 
and Marisela Gutierrez for recording NMR, MS, and IR spectra. DM thanks CONACYT for Graduate Scholarship.

\section{Notes and references}

1 G. Stork and L. A. Maldonado, J. Am. Chem. Soc., 1971, 93, 5286-5287.

2 R. J. H. Gregory, Chem. Rev., 1999, 99, 3649-3682.

3 J. D. Albright, Tetrahedron, 1983, 39, 3207-3233.

4 (a) G. Stork and L. Maldonado, J. Am. Chem. Soc., 1974, 96, 5273-5274; (b) G. Stork, J. C. Depezay and J. DAngelo, Tetrahedron Lett., 1975, 389-392; (c) J. Tsuji, J. Am. Chem. Soc., 1981, 103, 5259-5526; (d) G. Stork and T. Takahashi, J. Am. Chem. Soc., 1977, 99, 1275-1276; (e) G. Stork, T. Takahashi, I. Kawamoto and T. Suzuki, J. Am. Chem. Soc., 1978, 100, 8272-8273; (f) T. Takahashi, K. Kitamura, H. Nemoto, J. Tsuji and I. Miura, Tetrahedron Lett., 1983, 24, 3489-3492; $(g)$ T. Takahashi, H. Iwamoto, K. Nagashima, T. Okabe and T. Doi, Angew. Chem., Int. Ed., 1997, 36, 1319-1321; (h) O. S. Park, H. J. Hwang and W. Y. Lee, Arch. Pharmacal Res., 1993, 16, 205-208; (i) A. Kende, K. Liu, I. Kaldor, G. Dorey and K. Koch, J. Am. Chem. Soc., 1995, 117, 8258-8270; (j) I. Kadota, Y. Hu, G. K. Packard and S. D. Rychnovsky, Proc. Natl. Acad. Sci. U. S. A., 2004, 101(33), 11992-11995; (k) E. H. GranadosCovarrubias and L. A. Maldonado, J. Org. Chem., 2009, 74, 5097-5099.

5 (a) A. T. Au, Synth. Commun., 1984, 14, 743-748, For recent methodologies, see: (b) H. M. Torres-Domínguez, L. A. Maldonado and R. Le Lagadec, Tetrahedron Lett., 2020, 61, 151414-151417; (c) S. Aoki, S. Kotani, M. Sugiura and M. Nakajima, Tetrahedron Lett., 2010, 51, 3547-3549.

6 (a) L. F. Tietze and A. Modi, Med. Res. Rev., 2000, 20(4), 304322; (b) K. C. Nicolaou, D. J. Edmonds and P. G. Bulger, Angew. Chem., Int. Ed., 2006, 45, 7134-7186; (c) K. C. Nicolaou and J. S. Chen, Chem. Soc. Rev., 2009, 38, 2993-3009; (d) J. Poulin, C. M. Grisé-Bard and L. Barriault, Chem. Soc. Rev., 2009, 38, 3092-3101; (e) C. Grondal, M. Jeanty and D. Enders, Nat. Chem., 2010, 2, 167-178; $(f)$ H. Pellissier, Chem. Rev., 2013, 113(1), 442-524; (g) S. Zhi, S. Sanjun, X. Ma and W. Zhang, Org. Biomol. Chem., 2019, 17, 7632-7650.

7 H. M. Torres-Domínguez, L. A. Maldonado and R. Le Lagadec, Synth. Commun., 2017, 47, 1250-1255.

8 (a) A. Sharma, J. Pandey and R. P. Tripathi, Tetrahedron Lett., 2009, 50, 1812-1816; (b) T. Venkatesh, P. S. Mainkar and S. Chandrasekhar, J. Org. Chem., 2021, 86, 5412-5416; (c) K. Asahi and H. Nishino, Tetrahedron, 2008, 64, 1620-1634; (d) S. R. Lima and F. Coelho, ACS Omega, 2020, 5, 80328045 , and references therein.

9 (a) F. W. Sum and L. Weiler, Tetrahedron, 1981, 37, 303-317;

(b) B. Lygo, N. Oconnor and P. R. Wilson, Tetrahedron, 1988, 44, 688-6888; (c) S. Benetti, R. Romagnoli, C. De Risi, G. Spalluto and V. Zanirato, Chem. Rev., 1995, 95, 10651114; (d) C. Simon, C. Thierry and J. Jean Rodriguez, Eur. J. Org. Chem., 2004, 4957-4980; (e) D. Bonne, Y. Coquerel, T. Constantieux and J. Rodriguez, Tetrahedron: Asymmetry,
2010, 21, 1085-1109; $(f)$ T. Govender, P. I. Arvidsson, G. E. M. Maguire, H. G. Kruger and T. Naicker, Chem. Rev., 2016, 116, 9375-9437; (g) Y. S. Rao, Chem. Rev., 1976, 76, 625-694.

10 (a) V. Bhardwaj, D. Gumber, V. Abbot, S. Dhiman and P. Sharma, RSC Adv., 2015, 5, 15233-15266; (b) S. Khaghaninejad and M. M. Heravi, Adv. Heterocycl. Chem., 2014, 111, 95-146; (c) T. J. Donohoe and R. D. C. Pullin, Chem. Commun., 2012, 48, 11924-11938; (d) C. Schmuck and D. Rupprecht, Synthesis, 2007, 3095-3110.

11 S. P. Kolis, M. T. Clayton, J. L. Grutsch and M. M. Faul, Tetrahedron Lett., 2003, 44, 5707-5710.

12 B. Thapa and H. Schlegel, J. Phys. Chem. A, 2016, 120, 57265735.

13 J. D. Chai and M. Head-Gordon, Phys. Chem. Chem. Phys., 2008, 10, 6615-6620.

14 S. J. Grimme, Comput. Chem., 2006, 27, 1787-1799.

15 M. J. Frisch, G. W. Trucks, H. B. Schlegel, G. E. Scuseria, M. A. Robb, J. R. Cheeseman, G. Scalmani, V. Barone, G. A. Petersson, H. Nakatsuji, X. Li, M. Caricato, A. V. Marenich, J. Bloino, B. G. Janesko, R. Gomperts, B. Mennucci, H. P. Hratchian, J. V. Ortiz, A. F. Izmaylov, J. L. Sonnenberg, D. Williams-Young, F. Ding, F. Lipparini, F. Egidi, J. Goings, B. Peng, A. Petrone, T. Henderson, D. Ranasinghe, V. G. Zakrzewski, J. Gao, N. Rega, G. Zheng, W. Liang, M. Hada, M. Ehara, K. Toyota, R. Fukuda, J. Hasegawa, M. Ishida, T. Nakajima, Y. Honda, O. Kitao, H. Nakai, T. Vreven, K. Throssell, J. A. Montgomery, Jr, J. E. Peralta, F. Ogliaro, M. J. Bearpark, J. J. Heyd, E. N. Brothers, K. N. Kudin, V. N. Staroverov, T. A. Keith, R. Kobayashi, J. Normand, K. Raghavachari, A. P. Rendell, J. C. Burant, S. S. Iyengar, J. Tomasi, M. Cossi, J. M. Millam, M. Klene, C. Adamo, R. Cammi, J. W. Ochterski, R. L. Martin, K. Morokuma, O. Farkas, J. B. Foresman and D. J. Fox, Gaussian 16, Gaussian, Inc., Wallingford CT, 2016.

16 A. V. Marenich, C. J. Cramer and D. G. Truhlar, J. Phys. Chem. $B, 2009$, 113, 6378-6396.

17 (a) S. Dhers, A. Mondal, D. Aguilà, J. Ramírez, S. Vela, P. Dechambenoit, M. Rouzières, J. R. Nitschke, R. Clérac and J. M. Lehn, J. Am. Chem. Soc., 2018, 140, 8218-8227; (b) M. A. Rosero-Mafla, J. I. Castro, N. E. Sánchez, C. A. Mujica-Martinez and M. N. Chaur, ChemistrySelect, 2020, 5, 7685-7694.

18 (a) C. D. Johnson, The Hammett Equation, Cambridge University Press, Cambridge, 1973; (b) C. Hansch, A. Leo and R. Tatf, Chem. Rev., 1991, 91, 165-195.

19 A. Kuźnik, R. Mazurkiewicz and B. Fryczkowska, Beilstein J. Org. Chem., 2017, 13, 2710-2738.

20 A. Srikrishn and D. H. Dethe, Indian J. Chem., Sect. B: Org. Chem. Incl. Med. Chem., 2012, 51(2), 345-355.

21 P. Klahn, A. Duschek, C. Liébert and S. F. Kirsch, Org. Lett., 2012, 14, 1250-1253.

22 A. E. Cotman, B. Modec and B. Mohar, Org. Lett., 2018, 20, 2921-2924.

23 C. Zhang, H. Jiang and S. Zhu, Chem. Commun., 2017, 53, 2677-2680. 